\title{
Smoothness of the Density of States in the Anderson Model at High Disorder
}

\author{
Anton Bovier, Massimo Campanino ${ }^{\star}$, Abel Klein ${ }^{\star \star}$ and J. Fernando Perez ${ }^{\star \star \star}$ \\ Department of Mathematics, University of California, Irvine, CA 92717, USA
}

\begin{abstract}
We prove smoothness of the density of states in the Anderson model at high disorder for a class of potential distributions that include the uniform distribution.
\end{abstract}

\section{Introduction}

The Anderson model is given by the random Hamiltonian $H_{\varepsilon}=-\varepsilon / 2 \Delta+V$ on $l^{2}\left(\mathbf{Z}^{d}\right)$, where

$$
(\Delta u)(x)=\sum_{y \cdot|y-x|=1} u(y)
$$

and $V(x), x \in \mathbf{Z}^{d}$, are independent identically distributed random variables with common probability distribution $\mu$. The characteristic function of $\mu$ will be denoted by $h$, i.e., $h(t)=\int e^{-i t v} d \mu(v)$. The "disorder" is measured by $\varepsilon^{-1}, \varepsilon>0$.

If $\Lambda$ is a finite subset of $\mathbf{Z}^{d}$, we will denote by $H_{\varepsilon, \Lambda}$ the operator $H_{\varepsilon}$ restricted to $l^{2}(\Lambda)$ with zero boundary conditions outside $\Lambda$.

The integrated density of states, $N_{\varepsilon}(E)$, is defined by

$$
N_{\varepsilon}(E)=\lim _{\Lambda \rightarrow \mathbf{Z}^{d}}|\Lambda|^{-1} \#\left\{\text { eigenvalues of } H_{\varepsilon, \Lambda} \leqq E\right\} .
$$

It is a consequence of the ergodic theorem that for almost every potential the limit exists for all $E$ and is independent of the potential [1-4]. $N_{\varepsilon}(E)$ is always a continuous function [5-7], being log-Hölder continuous under mild conditions [6].

In one-dimension a lot is known about the integrated density of states. Under mild conditions it is always Hölder continuous on compact intervals $[8,9]$ and under some minimal regularity assumptions on $\mu$ it is differentiable, even infinitely differentiable [10-12].

* Permanent address: Dipartimento di Matematica, II Universita di Roma, Via Orazio Raimondo, I-00173 (La Romanina) Roma, Italy

$\star \star$ Research partially supported by the NSF under grants DMS 83-01889 and INT 85-03418

$\star \star \star$ Permanent address: Instituto de Fisica, Universidade de São Paulo, Caixa Postal 20516, São Paulo, S. P., Brazil. Research partially supported by the CNPq 
But in more than one dimension very little is known about the differentiability of $N_{\varepsilon}(E)$. There is an argument due to Edwards and Thouless [13] that shows that the density of states is analytic away from the edges of the spectrum if $\mu$ is the uniform distribution. If $\mu$ is absolutely continuous with respect to Lebesgue measured with a bounded density, Wegner [14] proved that $N_{\varepsilon}(E)$ is absolutely continuous with a bounded derivative. (See also [15].) Under the weaker hypothesis that $\mu$ is Hölder continuous, Carmona, Klein and Martinelli [9] obtained bounds on the differentiated density of states that suffice for the Fröhlich-MartinelliScoppola-Spencer method for proving localization.

Further results have required high disorder or low energy. Constantinescu, Fröhlich and Spencer [16] proved that if $\mu$ has a density analytic in a strip around the real axis, then the integrated density of states is analytic for $|E|$ large enough. If $\mu$ is Gaussian they proved that for high disorder $N_{\varepsilon}(E)$ is a real analytic function of $E$. Carmona [4], using an idea of Molcanov, has given a simple proof that if $h(t)$ is exponentially bounded, then $N_{\varepsilon}(t)$ is analytic at high disorder. Another simple argument for the same result due to Simon can be found in [16].

Differentiability results were obtained by Klein and Perez (unpublished). Using the supersymmetric replica trick and a cluster expansion Klein and Perez used the decay properties of $h(t)$ to derive differentiability for $N_{\varepsilon}(E)$ for high disorder or large $|E|$; their method also gave analyticity results. Their results for high disorder are:

Theorem 1.1. (i) Suppose $(1+t)^{d+n} h(t) \in L^{1}$, where $n \in\{0,1,2 \ldots\}$. Then there exists $\varepsilon_{0}>0$ such that $N_{\varepsilon}(E)$ is $(n+1)$-times continuously differentiable on the whole real line for all $0 \leqq \varepsilon<\varepsilon_{0}$.

(ii) Suppose $(1+t)^{d+n} h(t) \in L^{1}$ for all $n=0,1,2, \ldots$ Then there exists $\varepsilon_{0}>0$ such that $N_{\varepsilon}(E)$ is infinitely differentiable on the whole real line for all $0 \leqq \varepsilon<\varepsilon_{0}$.

(iii) Suppose $e^{-\alpha t} h(t)$ is bounded for some $\alpha>0$. Then for any $0<\alpha_{1}<\alpha$ there exists $\varepsilon_{1}=\varepsilon_{1}\left(\alpha_{1}\right)>0$ such that $N_{\varepsilon}(E)$ is analytic in the strip $|\operatorname{Im} E|<\alpha_{1}$ for all $0 \leqq \varepsilon<\varepsilon_{1}$.

In one dimension a similar result can be derived for any disorder by the methods of Campanino and Klein [11] as in their proof of Theorem 1.5, with the integrability condition on $(1+t)^{d+n} h(t)$ being replaced by the boundedness of that quantity.

In Theorem 1.1 as in the previous results for the multidimensional case (except for [15]), one gets out as much in regularity properties for $N_{\varepsilon}(E)$ as one puts in for the potential probability distribution $\mu$. Notice that the conclusions of Theorem 1.1 are valid for $\varepsilon=0$.

In this article we modify the methods of Klein and Perez to get out more than we put in, as done in [10-12] for the one-dimensional case. In particular we will obtain the infinite differentiability of $N_{\varepsilon}(E)$ for small $\varepsilon$ (but $\varepsilon \neq 0$; the result is not true for $\varepsilon=0$ ) if $\mu$ is the uniform distribution.

Our condition will be stated in terms of the characteristic function $h$ of the potential probability distribution $\mu$. We will only consider $h(t)$ for $t \geqq 0$ (of course, $h(-t)=\overline{h(t)})$ and differentiability at $t=0$ will mean right-hand side differentiability. Our result is 
Theorem 1.2. (i) Suppose $h$ is differentiable with $(1+t) h^{(i)}$ bounded, $i=0,1$. Then for any $0<E_{0}<\infty$ there exists $0<\varepsilon_{0}$ such that $N_{\varepsilon}(E)$ is continuously differentiable on $\left(-E_{0}, E_{0}\right)$ for $0<\varepsilon<\varepsilon_{0}$.

(ii) Suppose $h$ is $2 n$-times differentiable $(n \geqq 1)$ with $(1+t) h^{(i)}$ bounded $i=$ $0,1, \ldots, 2 n$. Then for any $0<E_{0}<\infty$ there exists $0<\varepsilon_{0}$ such that $N_{\varepsilon}(E)$ is $(n+1)$-times continuously differentiable on $\left(-E_{0}, E_{0}\right)$ for $0<\varepsilon<\varepsilon_{0}$.

(iii) Suppose $h$ is infinitely differentiable with $(1+t) h^{(i)}$ bounded, $i=0,1,2, \ldots$. Then for any $0<E_{0}<0$ there exists $0<\varepsilon_{0}$ such that $N_{\varepsilon}(E)$ is infinitely differentiable on $\left(-E_{0}, E_{0}\right)$ for $0<\varepsilon<\varepsilon_{0}$.

Corollary 1.3. Suppose $\mu$ is the uniform distribution. Then there exists $0<\varepsilon_{0}$ such that $N_{\varepsilon}(E)$ is infinitely differentiable on the whole real line for $0<\varepsilon<\varepsilon_{0}$.

We approach the density of states through the Green's function. Let

$$
G_{\varepsilon}(x, y ; z)=\left\langle x\left|\left(H_{\varepsilon}-z\right)^{-1}\right| y\right\rangle,
$$

where $x, y \in \mathbf{Z}^{d}, \operatorname{Im} z>0$. Then (e.g., $\left.[4,17]\right) G_{\varepsilon}(z)=\mathbf{E}\left(G_{\varepsilon}(0,0 ; z)\right)$ is the Borel transform of the measure $d N_{\varepsilon}(E)$, i.e.,

$$
G_{\varepsilon}(z)=\int \frac{d N_{\varepsilon}(E)}{E-z}
$$

and we have

i) $G_{\varepsilon}(E+i 0)=\lim G_{\varepsilon}(E+i \eta)$ exists for a.e. $E \in \mathbf{R}$.

ii) If $d N_{\varepsilon \text {,a.c. }}$ denotes the absolutely continuous part of the measure $d N_{\varepsilon}$, we have

$$
\frac{d N_{\varepsilon . \text { a.c. }}(E)}{d E}=\frac{1}{\pi} \operatorname{Im} G_{\varepsilon}(E+i 0) \text {. }
$$

iii) $d N_{\varepsilon, \text { sing }} \equiv d N_{\varepsilon}-d N_{\varepsilon \text {,a.c. }}$ is supported by the set

$$
\left\{E \in \mathbf{R} \mid \lim _{\eta \downarrow 0} \operatorname{Im} G_{\varepsilon}(E+i \eta)=\infty\right\} .
$$

Thus Theorems 1.1 and 1.2 will follows from

Theorem 1.4. (i) Suppose $(1+t)^{d+n} h(t) \in L^{1}$, where $n \in\{0,1,2, \ldots\}$. Then there exists $\varepsilon_{0}>0$ such that $G_{\varepsilon}(E+i 0)$ exists for all $E \in \mathbf{R}$ and is $n$-times continuously differentiable for all $0 \leqq \varepsilon<\varepsilon_{0}$.

(ii) Suppose $(1+t)^{d+n} h(t) \in L^{1}$ for all $n=0,1,2 \ldots$ Then there exists $\varepsilon_{0}>0$ such that $G_{\varepsilon}(E+i 0)$ exists for all $E \in \mathbf{R}$ and is infinitely differentiable for all $0 \leqq \varepsilon<\varepsilon_{0}$.

(iii) Suppose $e^{-\alpha t} h(t)$ is bounded for some $\alpha>0$. Then for any $0<\alpha_{1}<\alpha$ there exists $\varepsilon_{1}=\varepsilon_{1}\left(\alpha_{1}\right)>0$ such that $G_{\varepsilon}(z)$ has an analytic continuation to $\operatorname{Im} z>-\alpha_{1}$ for all $0 \leqq \varepsilon<\varepsilon_{1}$.

Theorem 1.5. (i) Suppose $h$ is differentiable with $(1+t) h^{(i)}$ bounded for $i=0,1$. Then for any $0<E_{0}<\infty$ there exists $\varepsilon_{0}>0$ such that $G_{\varepsilon}(E+i 0)$ exists and is continuous on the interval $\left(-E_{0}, E_{0}\right)$ for $0<\varepsilon<\varepsilon_{0}$.

(ii) Suppose $h$ is $2 n$-times differentiable $(n \geqq 1)$ with $(1+t) h^{(i)}$ bounded, $i=$ 
$0,1, \ldots, 2 n$. Then for any $0<E_{0}<\infty$ there exists $\varepsilon_{0}>0$ such that $G_{\varepsilon}(E+i 0)$ exists and is $n$-times continuously differentiable on the interval $\left(-E_{0}, E_{0}\right)$ for $0<\varepsilon<\varepsilon_{0}$.

(iii) Suppose $h$ is infinitely differentiable with $(1+t) h^{(i)}$ bounded for all $i=$ $0,1,2, \ldots$ Then for any $0<E_{0}<\infty$, there exists $\varepsilon_{0}>0$ such that $G_{\varepsilon}(E+i 0)$ exists and is infinitely differentiable on the interval $\left(-E_{0}, E_{0}\right)$ for $0<\varepsilon<\varepsilon_{0}$.

The strategy of our proofs will now be described. Let $\Lambda$ be a hypercube in $\mathbf{Z}^{d}$ centered at the origin, and let

$$
G_{\varepsilon, \Lambda}(z)=\mathbf{E}\left(\left\langle 0\left|\left(H_{\varepsilon, \Lambda}-z\right)^{-1}\right| 0\right\rangle\right) .
$$

We have $G_{\varepsilon}(z)=\lim _{|\Lambda| \rightarrow Z^{d}} G_{\varepsilon, \Lambda}(z)$ for $\operatorname{Im} z>0$.

We will use the supersymmetric replica trick $[18-21,11,22]$ to rewrite $G_{\varepsilon, \Lambda}(z)$ as a two-point function of a supersymmetric field theory. We will then perform a cluster expansion and do explicitly the integrations over the anticommuting variables. We will estimate the terms in the expansion and show convergence for small $\varepsilon$. This is the approach used by Klein and Perez and gives a proof of Theorem 1.4; this will be done in Sect. 2.

To prove Theorem 1.5 we will need to modify the cluster expansion. The assumptions of Theorem 1.5 do not give enough decay for the straightforward cluster expansion to converge. We will write $h=h_{1}+h_{2}$, where $h_{1}$ will have good decay properties. We will use a cluster expansion between sites equipped with $h_{1}$ and we will estimate the islands of $h_{2}$ 's taking oscillations into account. This is done in Sect. 3.

\section{A Supersymmetric Cluster Expansion}

2.1. The Supersymmetric Replica Trick. The supersymmetric replica trick says that

$$
G_{\varepsilon, \Lambda}\left(x_{1}, x_{2} ; z\right)=i \int \psi\left(x_{1}\right) \bar{\psi}\left(x_{2}\right) \exp \left\{-i \sum_{x, y \in \Lambda}\left\langle x\left|H_{\varepsilon, \Lambda}-z\right| y\right\rangle \Phi(x) \cdot \Phi(y)\right\} \mathscr{D}_{\Lambda} \Phi,
$$

where $\Lambda$ is a finite subset of $\mathbf{Z}^{d}, x_{1}, x_{2} \in \Lambda, \operatorname{Im} z>0, \Phi(x)=(\varphi(x), \psi(x), \bar{\psi}(x))$, where $\varphi(x) \in \mathbf{R}^{2}, \psi(x)$ and $\bar{\psi}(x)$ are anticommuting "variables" (i.e., elements of a Grassman algebra),

$$
\Phi(x) \cdot \Phi(y)=\varphi(x) \cdot \varphi(y)+\frac{1}{2}(\bar{\psi}(x) \psi(y)+\bar{\psi}(y) \psi(x))
$$

and $\quad \mathscr{D}_{\Lambda} \Phi=\prod_{x \in \Lambda} d \Phi(x)$, where $d \Phi(x)=(1 / \pi) d \bar{\psi}(x) d \psi(x) d^{2} \varphi(x)$. Notice that $\int e^{-\Phi(x) \cdot \Phi(x)} d \Phi(x)=1$.

Since we are working on a finite lattice $(2.1)$ is fully rigorous. To compute functions of $\psi, \bar{\psi}$ we expand in power series that terminate after a finite number of terms due to the anticommutativity. All $\{\psi(x), \bar{\psi}(x) ; x \in \Lambda\}$ anticommute. The linear functional denoted by integration against $d \bar{\psi}(x) d \psi(x)$ (it is not an actual integration) is defined by [23]

$$
\int\left(a_{0}+a_{1} \psi(x)+a_{2} \bar{\psi}(x)+a_{3} \bar{\psi}(x) \psi(x)\right) d \bar{\psi}(x) d \psi(x)=-a_{3} .
$$


To simplify our notation, we will abuse it by writing

$$
\Phi(x)^{2} \text { for } \Phi(x) \cdot \Phi(x) \text { and } \varphi(x)^{2} \text { for } \varphi(x) \cdot \varphi(x) \text {. }
$$

Recalling the definition of $H_{\varepsilon, \Lambda}$, we have

$$
\begin{aligned}
G_{\varepsilon, \Lambda}\left(x_{1}, x_{2} ; z\right)= & i \int \psi\left(x_{1}\right) \bar{\psi}\left(x_{2}\right) \exp \left\{-i \sum_{x \in \Lambda} V(x) \Phi(x)^{2}+i z \sum_{x \in \Lambda} \Phi(x)^{2}\right. \\
& \left.+i \varepsilon \sum_{\langle x, y\rangle \in \Lambda} \Phi(x) \cdot \Phi(y)\right\} \mathscr{D}_{\Lambda} \Phi,
\end{aligned}
$$

where by $\langle x, y\rangle \in \Lambda$ we denote a pair $x, y \in \Lambda$ with $|x-y|=1$, the summation being over all such pairs in $\Lambda$.

If $h$ is absolutely continuous with a bounded derivative, we can average over the random potential in (2.2) to obtain [11]

$$
\mathbf{E}\left(G_{\varepsilon, \Lambda}\left(x_{1}, x_{2} ; z\right)\right)=i \int \psi\left(x_{1}\right) \bar{\psi}\left(x_{2}\right) \prod_{x \in \Lambda} \beta\left(\Phi(x)^{2} ; z\right) \exp \left\{i \varepsilon \sum_{\langle x, y\rangle \in \Lambda} \Phi(x) \cdot \Phi(y)\right\} \mathscr{D}{ }_{\Lambda} \Phi,
$$

where $\beta(r ; z)=h(r) e^{i z r}$.

Thus

$$
G_{\varepsilon, \Lambda}(z)=i \int \psi(0) \bar{\psi}(0) \prod_{x \in \Lambda} \beta\left(\Phi(x)^{2} ; z\right) \exp \left\{i \varepsilon \sum_{\langle x, y\rangle \in \Lambda} \Phi(x) \cdot \Phi(y)\right\} \mathscr{D}_{\Lambda} \Phi .
$$

2.2. The Cluster Expansion. To perform a cluster expansion, we rewrite (2.3) as

$$
G_{\varepsilon, \Lambda}(z)=i \int \psi(0) \bar{\psi}(0) \prod_{x \in \Lambda} \beta\left(\Phi(x)^{2} ; z\right) \prod_{\langle x, y\rangle \in \Lambda}\left[\left(e^{i \varepsilon \Phi(x) \cdot \Phi(y)}-1\right)+1\right] \mathscr{D}{ }_{\Lambda} \Phi .
$$

Thus

$$
G_{\varepsilon, \Lambda}(z)=i \sum_{\Gamma \subset \Lambda} \int \psi(0) \bar{\psi}(0) \prod_{x \in \Lambda} \beta\left(\Phi(x)^{2} ; z\right) \prod_{\langle x, y\rangle \in \Gamma}\left(e^{i \varepsilon \Phi(x) \cdot \Phi(y)}-1\right) \mathscr{D}{ }_{\Lambda} \Phi,
$$

where by $\Gamma$ we denote a subset of nearest neighbor bonds. Notice that by $x \in \Gamma$ we mean that $x$ is a vertex in $\Gamma,\langle x, y\rangle \in \Gamma$ means the bond $\langle x, y\rangle$ is in $\Gamma$. We will denote by $b_{\Gamma}$ the number of bonds in $\Gamma$ and by $v_{\Gamma}$ the number of vertices in $\Gamma$. Notice that $v_{\Gamma} \leqq b_{\Gamma}+1$.

We now use the fact if $F\left(\Phi_{1}, \ldots, \Phi_{n}\right)$ is a supersymmetric function (with respect to the same supersymmetry acting on all the super-variables; see e.g., [24]), such that all of its components are integrable, then

$$
\int F\left(\Phi_{1}, \ldots, \Phi_{n}\right) d \Phi_{1} \ldots d \Phi_{n}=F(0, \ldots, 0) .
$$

This can be easily proved by induction. The case $n=1$ is just Lemma 4.3 in [24].

Using (2.5) on (2.4) we get (note that $\beta(0 ; z)=1$ )

$$
G_{\varepsilon, \Lambda}(z)=i \sum_{0 \in \Gamma \subset \Lambda} \int \psi(0) \bar{\psi}(0) \prod_{x \in \Gamma} \beta\left(\Phi(x)^{2} ; z\right) \prod_{\langle x, y\rangle \in \Gamma}\left(e^{i \varepsilon \Phi(x) \cdot \Phi(y)}-1\right) \mathscr{D}_{\Gamma} \Phi,
$$

where the sum is now only over connected graphs $\Gamma$ containing the origin.

We will now fix the connected graph $\Gamma, 0 \in \Gamma \subset \Lambda$, and perform the integration 
over the anticommuting variables. To do this notice that

$$
\begin{gathered}
\beta\left(\Phi^{2} ; z\right)=\beta\left(\varphi^{2} ; z\right)+\beta^{\prime}\left(\varphi^{2} ; z\right) \bar{\psi} \psi \\
e^{i \varepsilon \Phi_{1} \cdot \Phi_{2}}=e^{i \varepsilon \varphi_{1} \cdot \varphi_{2}}\left(1+\frac{i \varepsilon}{2}\left(\bar{\psi}_{1} \psi_{2}+\bar{\psi}_{2} \psi_{1}\right)+\frac{\varepsilon^{2}}{4} \bar{\psi}_{1} \psi_{1} \bar{\psi}_{2} \psi_{2}\right) .
\end{gathered}
$$

It follows that

$$
\int \psi(0) \bar{\psi}(0) \prod_{x \in \Gamma} \beta\left(\Phi(x)^{2} ; z\right) \prod_{\langle x, y\rangle \in \Gamma}\left(e^{i \varepsilon \Phi(x) \cdot \Phi(y)}-1\right) \mathscr{D} \Gamma^{\Phi}
$$

is a sum of terms of the form

$$
\pm \int \prod_{x \in \Gamma} \beta^{\# x}\left(\varphi(x)^{2} ; z\right) \prod_{\langle x, y\rangle \in \Gamma} \$(x, y)(\varphi(x), \varphi(y)) \mathscr{D}_{\Gamma} \varphi
$$

where $\beta^{\#_{x}}$ is either $\beta$ or $\beta^{\prime}$, and $\$(x, y)(\varphi(x), \varphi(x))$ can be either $\left(e^{i \varepsilon \varphi(x) \cdot \varphi(y)}-1\right)$, $(i \varepsilon / 2) e^{i \varepsilon \varphi(x) \cdot \varphi(y)}$ or $\left(\varepsilon^{2} / 4\right) e^{i \varepsilon \varphi(x) \cdot \varphi(y)}$ and $\mathscr{D}_{\Gamma} \varphi=\prod_{x \in \Gamma} d^{2} \varphi(x) / \pi$.

If in Theorem 1.4 we had also made assumptions on the derivatives of $h$ similar to the ones made on $h$, we could now estimate each term in (2.9) to prove a version of Theorem 1.4 that would look more like Theorem 1.5. In particular our choice of $\varepsilon_{0}$ would depend on the energy interval $\left(-E_{0}, E_{0}\right)$.

To avoid the assumptions on the derivatives and the dependency of $\varepsilon_{0}$ on the energy interval, let us look again at (2.9). Notice that if $\beta^{\#_{x}}=\beta^{\prime}$, then it follows from the integration over the anticommuting variables that we must have $\$(x, y)(\varphi(x), \varphi(y))=e^{i \varepsilon \varphi(x) \cdot \varphi(y)}-1$ if $\langle x, y\rangle \in \Gamma$. We will exploit this fact when performing an integration by parts on the variable $\varphi(x)$ to get rid of the derivative. We have

$$
\beta^{\prime}\left(\varphi^{2}\right)=\left(2 \varphi^{2}\right)^{-1} \varphi \cdot \nabla \beta\left(\varphi^{2}\right) .
$$

Thus, with $\operatorname{Im} z>0$ (we omit $z$ ),

$$
\begin{aligned}
\int \beta^{\prime}\left(\varphi^{2}\right) \prod_{j=1}^{k}\left(e^{i \varepsilon \varphi \cdot \varphi_{J}}-1\right) d^{2} \varphi & =-\int \beta\left(\varphi^{2}\right) \nabla \cdot\left[\left(2 \varphi^{2}\right)^{-1} \varphi \prod_{j=1}^{k}\left(e^{i \varepsilon \varphi \cdot \varphi_{J}}-1\right)\right] d^{2} \varphi \\
& =-\int \beta\left(\varphi^{2}\right)\left(2 \varphi^{2}\right)^{-1} \varphi \cdot \nabla \prod_{j=1}^{k}\left(e^{i \varepsilon \varphi \cdot \varphi_{J}}-1\right) d^{2} \varphi \\
& =-\int \beta\left(\varphi^{2}\right) \sum_{j=1}^{k} i \varepsilon\left(2 \varphi^{2}\right)^{-1} \varphi \cdot \varphi_{j} e^{i \varepsilon \varphi \cdot \varphi_{j}} \prod_{l \neq j}\left(e^{i \varepsilon \varphi \cdot \varphi_{l}}-1\right) d^{2} \varphi,
\end{aligned}
$$

since $\nabla \cdot\left(2 \varphi^{2}\right)^{-1} \varphi=\delta(\varphi)$, and $\beta\left(\varphi^{2}\right) \prod_{j=1}^{k}\left(e^{i \varepsilon \varphi \cdot \varphi_{J}}-1\right)$ is continuous and equal to zero at $\varphi=0$.

We may also have to do an integration by parts when we had already done an integration by parts on one side of a bond. In this case, we have

$$
\begin{aligned}
& \int \beta^{\prime}\left(\varphi^{2}\right) i \varepsilon \varphi \cdot \varphi_{1}\left(2 \varphi_{1}\right)^{-2} e^{i \varepsilon \varphi \cdot \varphi_{1}} d^{2} \varphi \\
& \quad=-i \varepsilon \int \beta\left(\varphi^{2}\right)\left(2 \varphi^{2}\right)^{-1} \varphi \cdot \nabla\left(\varphi \cdot \varphi_{1}\left(2 \varphi_{1}^{2}\right)^{-1} e^{i \varepsilon \varphi \cdot \varphi_{1}}\right) d^{2} \varphi \\
& \quad=-i \varepsilon \int \beta\left(\varphi^{2}\right)\left[\left(4 \varphi^{2} \varphi_{1}^{2}\right)^{-1} \varphi \cdot \varphi_{1}+i \varepsilon\left(4 \varphi^{2} \varphi_{1}^{2}\right)^{-1}\left(\varphi \cdot \varphi_{1}\right)^{2}\right] e^{i \varepsilon \varphi \cdot \varphi_{1}} d^{2} \varphi .
\end{aligned}
$$


Thus each term in (2.9) can be written as the sum of at most $(2 d)^{v} \Gamma$ terms of the same form except that $\beta^{\#_{x}}$ is always $\beta$ and $\$(x, y)(\varphi(x), \varphi(y))$ can also be

$$
i \varepsilon\left(2 \varphi(x)^{2}\right)^{-1} \varphi(x) \cdot \varphi(y) e^{i \varepsilon \varphi(x) \cdot \varphi(y)},
$$

or

$$
i \varepsilon \varphi(x) \cdot \varphi(y)\left(2 \varphi(y)^{2}\right)^{-1} e^{i \varepsilon \varphi(x) \cdot \varphi(y)}
$$

or

$$
-i \varepsilon\left[4 \varphi(x)^{2} \varphi(y)^{2}\right]^{-1} \varphi(x) \cdot \varphi(y) e^{i \varepsilon \varphi(x) \cdot \varphi(y)}(1+i \varepsilon \varphi(x) \cdot \varphi(y)) .
$$

In any case, we always have

$$
\begin{aligned}
|\$(x, y)(\varphi(x), \varphi(y))| & \leqq \varepsilon(1+|\varphi(x)|)(1+|\varphi(y)|) \\
& \text { or } \leqq \varepsilon(1+|\varphi(x)|)(2|\varphi(y)|)^{-1} \\
& \text { or } \leqq \varepsilon\left(1+(|\varphi(y)|)(2|\varphi(x)|)^{-1}\right. \\
& \text { or } \leqq \varepsilon\left(1+(2|\varphi(x)|)^{-1}\right)\left(1+(2|\varphi(y)|)^{-1}\right)
\end{aligned}
$$

with the important restriction that for a given $x$ at most one of the bonds for which $x$ is a vertex contributes a $(2|\varphi(x)|)^{-1}$ factor.

Thus each term in (2.9) can be bounded by $\varepsilon^{b} \Gamma(2 d)^{v} \Gamma C_{1}^{v} \Gamma$, where $C_{1}$ is the biggest of

$$
\int(1+|\varphi|)^{2 d}\left|\beta\left(\varphi^{2} ; z\right)\right| \frac{d^{2} \varphi}{\pi}
$$

or

$$
\int(1+|\varphi|)^{2 d-1}(2|\varphi|)^{-1}\left|\beta\left(\varphi^{2} ; z\right)\right| \frac{d^{2} \varphi}{\pi} .
$$

Since $\left|\beta\left(\varphi^{2} ; z\right)\right| \leqq\left|h\left(\varphi^{2}\right)\right|, C_{1}$ can be chosen independently of $z$. Notice that $C_{1}<\infty$ by the hypothesis of Theorem 1.4 .

Since the number of terms in $(2.9)$ is $\leqq 2^{v} \Gamma 3^{b} \Gamma$, we have that

$$
\begin{gathered}
\left|\int \psi(0) \bar{\psi}(0) \prod_{x \in \Gamma} \beta\left(\phi(x)^{2} ; z\right) \prod_{\langle x, y\rangle \in \Gamma}\left(e^{i \varepsilon \Phi(x) \cdot \Phi(y)}-1\right) \mathscr{D}_{\Gamma} \Phi\right| \\
\leqq \varepsilon^{b} \Gamma\left(2 d+C_{1}+2\right)^{v} \Gamma 3^{b_{I}} \leqq C_{2}^{b_{\Gamma}+1} \varepsilon^{b_{\Gamma}}
\end{gathered}
$$

for $C_{2}=\left(2 d+C_{1}+2\right) 3$

It now follows from (2.6) that

$$
\begin{aligned}
\left|G_{\varepsilon, \Lambda}(z)\right| & \leqq \sum_{0 \in \Gamma \subset \Lambda} C_{2}^{\left(1+b_{\Gamma}\right)} \varepsilon^{b} \Gamma=\sum_{n=0}^{\infty} \sum_{0 \in \Gamma \subset \Lambda \text { with } b_{\Gamma}=n} C_{2}^{(1+n)} \varepsilon^{n} \\
& \leqq C_{2} \sum_{n=0}^{\infty}\left(4 d^{2} C_{2} \varepsilon\right)^{n}=C_{2}\left(1-2 d C_{2} \varepsilon\right)^{-1}
\end{aligned}
$$

if

$$
\varepsilon<\left(4 d^{2} C_{2}\right)^{-1}
$$

Under the above assumptions $G_{\varepsilon, \Lambda}(E+i \eta)$ can be extended to $\eta=0$ as a 
continuous function. Since all our bounds are uniform in $E \in \mathbf{R}$ and $\eta \geqq 0$ we can conclude that $G_{\varepsilon, \Lambda}(E+i \eta)$ converges as $\Lambda \rightarrow \mathbf{Z}^{d}$ uniformly on $E \in \mathbf{R}$ and $\eta \geqq 0$. Since for $\eta>0$ the convergence is to $G_{\varepsilon}(E+i \eta)$, we can conclude that $G_{\varepsilon}(E+i 0)$ exists and is a continuous function on the whole real line.

We now turn to the differentiability of $G_{\varepsilon}(E+i 0)$ with respect to $E$. Since $\beta\left(\Phi^{2} ; z\right)=h\left(\Phi^{2}\right) e^{i z \Phi^{2}}$, it is clear from $(2.3)$ that $G_{\varepsilon, \Lambda}(z)$ can be differentiated with respect to $E$ for $\eta \geqq 0$. If we use (2.4) we get

$$
\frac{d}{d E} G_{\varepsilon, \Lambda}(z)=-\sum_{0 \in \Gamma \subset \Lambda} \sum_{x \in \Gamma} \int \psi(0) \bar{\psi}(0) \Phi(x)^{2} \prod_{x \in \Gamma} \beta\left(\Phi(x)^{2} ; z\right) \prod_{\langle x, y\rangle \in \Gamma}\left(e^{i \varepsilon \Phi(x) \cdot \Phi(y)}-1\right) \mathscr{D}_{\Gamma} \Phi .
$$

As before each term in the sum can be bounded by $C_{3} C_{2}^{b} \Sigma_{\varepsilon}^{b}{ }^{b}$, where $C_{3}$ is calculated like $C_{1}$ except that we replace $\beta$ in (2.13) by $\varphi^{2} \beta$. Notice that $C_{3}<\infty$ is the hypothesis of Theorem 1.4 for the differentiability of $G_{\varepsilon}(E+i 0)$.

Thus we have the bond

$$
\left|\frac{d}{d E} G_{\varepsilon, \Lambda}(z)\right| \leqq \sum_{0 \in \Gamma \subset \Lambda} v_{\Gamma} C_{3} C_{2}^{b_{\Gamma}} \varepsilon^{b_{\Gamma}} \leqq C_{3} \sum_{n=0}^{\infty}(1+n)\left(4 d^{2} C_{2} \varepsilon\right)^{n}<\infty
$$

if, as before, $\varepsilon<\left(4 d^{2} C_{2}\right)^{-1}$.

The same procedure works for higher derivatives.

Since $h^{\prime}$ does not appear in our bounds we can remove it by an approximation argument. This concludes the proof of Theorem 1.4 (i) and (ii). To prove the analyticity in (iii) the same procedure works since $G_{\varepsilon, \Lambda}(z)$ is analytic for $\operatorname{Im} z>-\alpha$. One proves uniform bounds for $\operatorname{Im} z \geqq-\alpha_{1}$ and uses Vitali's Theorem.

\section{The Modified Cluster Expansion}

3.1. Basic Idea. We will now modify the cluster expansion of Sect. 2 to prove Theorem 1.5. For technical reasons we will use the supersymmetric replica trick for $\varepsilon^{-1} H_{\varepsilon}=-\frac{1}{2} \Delta+\varepsilon^{-1} V$. Thus (2.3) is rewritten as

$$
G_{\varepsilon, \Lambda}(z)=i \varepsilon^{-1} \int \psi(0) \bar{\psi}(0) \prod_{x \in \Lambda} \beta_{\varepsilon}\left(\Phi^{2}(x) ; z\right) \exp \left\{i \sum_{\langle x, y\rangle \in \Lambda} \Phi(x) \cdot \Phi(y)\right\} \mathscr{D}_{\Lambda} \Phi,
$$

where $\beta_{\varepsilon}(r ; z)=\beta\left(\varepsilon^{-1} r ; z\right)$.

We will write $\beta_{\varepsilon}=\beta_{1}+\beta_{2}$, the decomposition depending on $\varepsilon . \beta_{1}$ will be chosen to have compact support and $\beta_{2}$ will be the tail. Our approach will be to perform a cluster expansion only on bonds between lattice sites equipped with $\beta_{1}$, and choose $\beta_{1}$ and $\beta_{2}$ in such a way that the integrals over the regions equipped with $\beta_{2}$ are small. The convergence of the expansion will then depend on a delicate balancing of bounds.

3.2. The Decomposition of $\beta_{\varepsilon}$ and Bounds. Let $\chi$ be a fixed infinitely differentiable function on $\mathbf{R}$ such that $0 \leqq \chi \leqq 1, \chi \equiv 1$ on $\left[-\frac{1}{2}, \frac{1}{2}\right]$ and $\operatorname{supp} \chi \subset[-1,1]$. We will use $\tilde{\chi}$ to denote the characteristic function of $[-1,1]$.

We now fix $\varepsilon>0$ and define (we omit the complex energy $z$ )

$$
\beta_{1}(t)=\beta_{\varepsilon}(t) \chi\left(\varepsilon^{-\delta} t\right), \quad \beta_{2}(t)=\beta_{\varepsilon}(t)-\beta_{1}(t)=\beta_{\varepsilon}(t)\left(1-\chi\left(\varepsilon^{-\delta} t\right)\right) .
$$


Here $0<\delta<1$ is to be chosen later. Our assumptions are that

$$
\left|h^{(i)}(t)\right| \leqq C(1+t)^{-1} \text { for } i=0,1 .
$$

By $C$ we will always denote an appropriate finite constant (not always the same).

We will derive bounds on $\beta_{1}$ and $\beta_{2}$ that will be needed for the convergence of the modified cluster expansion. We now fix $0<E_{0}<\infty$. All our bounds will be uniform in $E$ and $\eta$ for $|E|<E_{0}$ and $0 \leqq \eta \leqq 1$.

Notice that

$$
\int_{0}^{\infty}(1+t)^{-1} \chi\left(\varepsilon^{1-\delta} t\right) d t \leqq \int_{0}^{\varepsilon^{-1+\delta}}(1+t)^{-1} d t=\log \left(1+\varepsilon^{-1+\delta}\right) \leqq C|\log \varepsilon|
$$

and

$$
\int_{0}^{\infty}(1+t)^{-2}\left(1-\chi\left(\varepsilon^{1-\delta} t\right)\right)^{2} d t \leqq \int_{1 / 2 \varepsilon}^{\infty} \frac{-1+\delta}{1+t}(1)^{-2} d t=\left(1+\frac{1}{2} \varepsilon^{-1+\delta}\right)^{-1} \leqq C \varepsilon^{1-\delta} .
$$

From now on we will always consider $\beta_{1}, \beta_{2}, h, h^{\prime}$, etc. as functions of $\varphi^{2}$, and all the $L^{p}$ norms will always be with respect to $d^{2} \varphi$.

From (3.2), (3.3), (3.4), and (3.5) we get:

$$
\begin{aligned}
&\left\|\beta_{1}\right\|_{1} \leqq C \varepsilon|\log \varepsilon|, \\
&\left\|\beta_{1}^{\prime}\right\|_{1} \leqq C|\log \varepsilon|, \leqq C \varepsilon^{1 / 2} \\
&\left\|\beta_{1}\right\|_{2} \leqq C \varepsilon^{-1 / 2} \\
&\left\|\beta_{1}^{\prime}\right\|_{2} \leqq \varepsilon_{2} \|_{2} \leqq C \varepsilon^{1-\delta / 2} \\
&\left\|\beta_{2}^{\prime}\right\|_{2} \leqq C \varepsilon^{-\delta / 2} \\
&\left\||\varphi|^{-1} \beta_{2}\right\|_{2} \leqq C \varepsilon^{1-\delta} \\
&\left\||\varphi| \beta_{2}\right\|_{\infty} \leqq C \varepsilon^{1-\delta / 2} \\
&\left\||\varphi| \beta_{2}^{\prime}\right\|_{\infty} \leqq C \varepsilon^{-\delta / 2} \\
&\left\||\varphi| \beta_{1}\right\|_{2} \leqq C \varepsilon|\log \varepsilon|^{1 / 2} \\
&\left\||\varphi| \beta_{1}^{\prime}\right\|_{2} C|\log \varepsilon|^{1 / 2}
\end{aligned}
$$

For $1<p<\infty$ we have

$$
\begin{aligned}
&\left\|\beta_{1}\right\|_{p} \leqq C \varepsilon^{1 / p} \\
&\left\|\beta_{1}^{\prime}\right\|_{p} \leqq C \varepsilon^{-1+1 / p} \\
&\left\|\beta_{2}\right\|_{p} \leqq C \varepsilon^{1-(1-1 / p) \delta} \\
&\left\|\beta_{2}^{\prime}\right\|_{p} \leqq C \varepsilon^{-(1-1 / p) \delta}
\end{aligned}
$$

For $1 \leqq p<2$ we have

$$
\left\||\varphi| \beta_{1}\right\|_{p} \leqq C \varepsilon^{1+\delta(1 / p-1 / 2)}
$$




$$
\left\||\varphi| \beta_{1}\right\|_{p} \leqq C \varepsilon^{\delta(1 / p-1 / 2)}
$$

For $\frac{2}{3}<p<\infty$ we have

$$
\left\||\varphi|^{-1} \beta_{2}\right\|_{p} \leqq C \varepsilon^{1-\delta(3 / 2-1 / p)} .
$$

For $2<p<\infty$ we have

$$
\begin{gathered}
\left\||\varphi| \beta_{2}\right\|_{p} \leqq C \varepsilon^{1-\delta(1 / 2-1 / p)} \\
\left\||\varphi| \beta_{2}^{\prime}\right\|_{p} \leqq C \varepsilon^{-\delta(1 / 2-1 / p)} .
\end{gathered}
$$

Another bound we will need is

$$
\left\|\left(e^{i \varphi \cdot \varphi^{\prime}}-1\right) \tilde{\chi}\left(\varepsilon^{-\delta} \varphi^{2}\right) \tilde{\chi}\left(\varepsilon^{-\delta} \varphi^{\prime 2}\right)\right\|_{\infty} \leqq C \varepsilon^{\delta} .
$$

3.3. The Modified Expansion. From (3.1) and (3.2) we have

$$
\begin{aligned}
-i \varepsilon G_{\varepsilon, \Lambda}(z) & =\sum_{\substack{k(x)=1,2 \\
x \in \Lambda}} \int \psi(0) \bar{\psi}(0) \prod_{x \in \Lambda} \beta_{k(x)}\left(\Phi(x)^{2} ; z\right) \exp \left\{i \sum_{\langle x, y\rangle \in \Lambda} \Phi(x) \cdot \Phi(y)\right\} \mathscr{D}_{\Lambda} \Phi \\
& =\sum_{\Lambda_{1} \subset \Lambda} \int \psi(0) \bar{\psi}(0) \prod_{x \in \Lambda_{1}} \beta_{1}\left(\Phi(x)^{2}\right) \prod_{x \in \Lambda \backslash \Lambda_{1}} \beta_{2}\left(\Phi(x)^{2}\right) \prod_{\langle x, y\rangle \in \Lambda} e^{i \Phi(x) \cdot \Phi(y)} \mathscr{D}_{\Lambda} \Phi .
\end{aligned}
$$

We will perform a cluster expansion only on bonds between sites in $\Lambda_{1}$. For a fixed $\Lambda_{1} \subset \Lambda$ we let $\Lambda_{2}=\Lambda \backslash \Lambda_{1}$,

$$
\bar{\Lambda}_{2}=\left\{x \in \Lambda \mid d\left(x_{1}, \Lambda_{2}\right) \leqq 1\right\}, \quad \mathscr{T}\left(\Lambda_{1}\right)=\left\{\langle x, y\rangle \in \Lambda_{1} \mid\langle x, y\rangle \notin \bar{\Lambda}_{2}\right\} .
$$

To perform the cluster expansion we rewrite (3.27) as

$$
\begin{aligned}
-i \varepsilon G_{\varepsilon, \Lambda}(z)= & \sum_{\Lambda_{1} \subset \Lambda} \int \psi(0) \bar{\psi}(0) \prod_{x \in \Lambda_{1}} \beta_{1}\left(\Phi(x)^{2}\right) \prod_{x \in \Lambda_{2}} \beta_{2}\left(\Phi(x)^{2}\right) \\
& \cdot \prod_{\langle x, y\rangle \in \mathscr{T}\left(\Lambda_{1}\right)}\left[\left(e^{i \Phi(x) \cdot \Phi(y)}-1\right)+1\right] \prod_{\langle x, y\rangle \notin \mathscr{T}\left(\Lambda_{1}\right)} e^{i \Phi(x) \cdot \Phi(y) \mathscr{D}_{\Lambda} \Phi} \\
= & \sum_{\Lambda_{1} \subset \Lambda} \sum_{\Gamma \subset \mathscr{T}\left(\Lambda_{1}\right)} \int \psi(0) \bar{\psi}(0) \prod_{x \in \Lambda_{1}} \beta_{1}\left(\Phi(x)^{2}\right) \prod_{x \in \Lambda_{2}} \beta_{2}\left(\Phi(x)^{2}\right) \\
& \cdot \prod_{\langle x, y\rangle \in \Gamma}\left(e^{i \Phi(x) \cdot \Phi(y)}-1\right) \prod_{\langle x, y\rangle \notin \mathscr{T}\left(\Lambda_{1}\right)} e^{i \Phi(x) \cdot \Phi(y) \mathscr{D}_{\Lambda} \Phi .}
\end{aligned}
$$

We now use (2.5) and the discussion before it. Taking into account that $\beta_{1}(0)=1$ and $\beta_{2}(0)=0$, we get

$$
\begin{aligned}
-i \varepsilon G_{\varepsilon, \Lambda}(z)= & \sum_{\Lambda_{2} \subset \Lambda} \sum_{\Gamma \in \mathscr{G}\left(\Lambda_{2}\right)} \int \psi(0) \bar{\psi}(0) \prod_{x \in \Lambda_{2}} \beta_{2}\left(\Phi(x)^{2}\right) \\
& \cdot \prod_{x \in\left[\left(\bar{\Lambda}_{2} \cup \Gamma\right) \backslash \Lambda_{2}\right]} \beta_{1}\left(\Phi(x)^{2}\right) \prod_{\langle x, y\rangle \in \Gamma}\left(e^{i \Phi(x) \cdot \Phi(y)}-1\right) \\
& \cdot \prod_{\langle x, y\rangle \in \bar{\Lambda}_{2}} e^{i \Phi(x) \cdot \Phi(y)} \mathscr{D}_{\Gamma \cup \bar{\Lambda}_{2}} \Phi,
\end{aligned}
$$

where $\mathscr{G}\left(\Lambda_{2}\right)=\left\{\Gamma \in \mathscr{T}\left(\Lambda_{1}\right) \mid \Gamma \cup \bar{\Lambda}_{2}\right.$ is a connected set of nearest neighbors bonds with $\left.0 \in \Gamma \cup \bar{\Lambda}_{2}\right\}$.

We now perform the integration over the anticommuting variables. If we fix $\Lambda_{2} \subset \Lambda, \Gamma \in \mathscr{G}\left(\Lambda_{2}\right)$, the corresponding term in (3.28) can be written as a sum of 
terms of the form (recall (2.7) and (2.8))

$$
\begin{aligned}
\pm \int & \prod_{x \in \Lambda_{2}} \beta_{2}^{\#_{x}}\left(\varphi(x)^{2}\right) \prod_{\substack{x \in\left[\left(\Gamma \cup \bar{\Lambda}_{2}\right) \backslash \Lambda_{2}\right]\\
}} \beta_{1}^{\#_{x}}\left(\varphi(x)^{2}\right) \\
& \cdot \prod_{\langle x, y\rangle \in \Gamma} \$(x, y)\left(e^{i \varphi(x) \cdot \varphi(y)}-b(x, y)\right) \prod_{\langle x, y\rangle \in \bar{\Lambda}_{2}} \$(x, y) e^{i \varphi(x) \cdot \varphi(y)} \mathscr{D}_{\Gamma \cup \bar{X}_{2}} \varphi,
\end{aligned}
$$

where

$$
\begin{aligned}
& \beta_{i}^{\#_{x}} \text { is either } \beta_{i} \text { or } \beta_{i}^{\prime}, i=1,2, \\
& b(x, y) \text { is either } 0 \text { or } 1 \\
& \$(x, y) \text { is either } 1, \frac{i}{2} \text { or } \frac{1}{4}
\end{aligned}
$$

There are constraints on the possible choices in (3.30). We are not going to take these constraints into account except for the following: if at a given site $x$ we have $\beta_{1}^{\prime}\left(\varphi(x)^{2}\right)$ then we have $b(x, y)=1$ for all $\langle x, y\rangle \in \Gamma$.

3.4. Integration by Parts. The $L^{p}$-bounds (3.11) and (3.20) for $\beta_{2}^{\prime}$ diverge like $\varepsilon^{-\delta}$ with $\delta>0$. If we have a term in (3.29) with many $\beta_{2}^{\prime}$, our estimate will give us a large factor. To avoid this problem we will thin out the number of $\beta_{2}^{\prime}$ by performing integration by parts.

So let us fix a term in (3.29) and write $\Lambda_{2}=B \cup B^{\prime}$, where $B$ is the subset of $\Lambda_{2}$ where we have $\beta_{2}$, and $B^{\prime}$ is the subset of $\Lambda_{2}$ where we have $\beta_{2}^{\prime}$. We want to perform an integration by parts in a sufficiently large subset $B^{\prime \prime}$ of $B^{\prime}$ such that the sites in $B^{\prime \prime}$ are sufficiently far apart from each other.

Notice that

$$
\mathbf{Z}^{d}=\bigcup_{\alpha}\left(7 \mathbf{Z}^{d}+\alpha\right)
$$

the union being over all $\alpha \in \mathbf{Z}^{d}$ with $\alpha_{i} \in\{0,1,2,3,4,5,6\}, i=1,2, \ldots, d$.

Since (3.31) expresses $\mathbf{Z}^{d}$ as a disjoint union, there exists at least one such $\alpha$, say $\tilde{\alpha}$, such that

$$
\left|B^{\prime} \cap\left(7 \mathbf{Z}^{d}+\tilde{\alpha}\right)\right| \geqq 7^{-d}\left|B^{\prime}\right|
$$

We choose

$$
B^{\prime \prime}=B^{\prime} \cap\left(7 \mathbf{Z}^{d}+\tilde{\alpha}\right) .
$$

For $x \in B^{\prime \prime}$ we perform an integration by parts similar to (2.11). Since $\beta_{2}=0$ in a neighborhood of the origin we get

$$
\begin{aligned}
& \int \beta_{2}^{\prime}\left(\varphi(x)^{2}\right) \exp \left\{i \varphi(x) \cdot \sum_{y:\langle x, y\rangle \in \bar{\Lambda}_{2}} \varphi(y)\right\} d^{2} \varphi(x) \\
& =-i \sum_{y:\langle x, y\rangle \in \bar{\Lambda}_{2}} \int \beta_{2}\left(\varphi(x)^{2}\right)\left[2 \varphi(x)^{2}\right]^{-1} \varphi(x) \cdot \varphi(y) \\
& \quad \cdot \exp \left\{i \varphi(x) \cdot \sum_{y:\langle x, y\rangle \in \bar{\Lambda}_{2}} \varphi(y)\right\} d^{2} \varphi(x)
\end{aligned}
$$




$$
\begin{aligned}
= & -i \sum_{y \cdot\langle x, y\rangle \in \bar{\Lambda}_{2}} \sum_{j=1}^{2} \int \beta_{2}\left(\varphi(x)^{2}\right)\left[2 \varphi(x)^{2}\right]^{-1} \varphi_{j}(x) \varphi_{j}(y) \\
& \cdot \exp \left\{i \varphi(x) \cdot \sum_{y:\langle x, y\rangle \in \bar{\Lambda}_{2}} \varphi(y)\right\} d^{2} \varphi(x) .
\end{aligned}
$$

After performing the integration by parts (3.34) at all sites in $B^{\prime \prime}$, we can still express each term in (3.28) as a sum of terms of the form given by (3.29), except that now

$\beta_{1}^{\#_{x}}$ is either $\beta_{1}$ or $\beta_{1}^{\prime}$ if $x \in \Gamma \backslash \bar{\Lambda}_{2}$,

or $\beta_{1}, \beta_{1}^{\prime}, \varphi_{j} \beta_{1}, \varphi_{j} \beta_{1}^{\prime}, j=1,2$, if $x \in \Gamma \cap \bar{\Lambda}_{2}$;

$\beta_{2}^{\#_{x}}$ is either $\beta_{2}, \beta_{2}^{\prime}, \varphi_{j} \beta_{2}, \varphi_{j} \beta_{2}^{\prime}$,

are as in (3.30)

or $\left[2 \varphi^{2}\right]^{-1} \varphi_{j} \beta_{2}, j=1,2, b(x, y)$ and $\$(x, y)$

3.5. Convergence of the Expansion. Let us fix a term of the form (3.29) with the choices for the functions in the integrand being done according to (3.35). We are going first to integrate over the variables in each connected component of $\bar{\Lambda}_{2}$. The functions at each site of $\Lambda_{2}$ are not in $L^{1}$ except for those at the sites in $B^{\prime \prime}$ where an integration by parts has been performed, but they are in all $L^{p}$ for $p>1$ with the possible exception of one of the nearest neighbors of each site in $B^{\prime \prime}$ where we need $p>2$. We must use the oscillations given by the bond functions in order to be able to estimate the integrals. This will be achieved by interpolating between different estimates using $L^{p}$ norms.

The crucial estimate is given by the following lemma:

Lemma 3.1. Let

$$
\begin{aligned}
K= & K\left(\Lambda_{2}, \Gamma, \#, \$, b\right)=\int \prod_{x \in \bar{\Lambda}_{2} \backslash \Lambda_{2}} \beta_{1}^{\#_{x}}\left(\varphi(x)^{2}\right) \prod_{x \in \Lambda_{2}} \beta_{2}^{\# x}\left(\varphi(x)^{2}\right) \prod_{\langle x, y\rangle \in \bar{\Lambda}_{2}} \$(x, y) e^{i \varphi(x) \cdot \varphi(y)} \\
& \cdot \prod_{\langle x, w\rangle \in \Gamma, x \in \bar{\Lambda}_{2}}\left[\$(x, w)\left(e^{i \varphi(x) \cdot \varphi(w)}-b(x, w)\right) \tilde{\chi}\left(\varepsilon^{-\delta} \varphi(x)^{2}\right) \tilde{\chi}\left(\varepsilon^{-\delta} \varphi(w)^{2}\right)\right] \mathscr{D}_{\bar{\Lambda}_{2}} \varphi .
\end{aligned}
$$

Then there are $1<p<2,2<r<\infty, 1<q=q(r)<\infty$ with $\lim _{r \rightarrow 2} q(r)=1$, and $a$ constant $C=C(p, q, r)<\infty$, all independent of $\Lambda_{2}, \Gamma, \#, \$, b$, such that

$$
\begin{aligned}
|K| \leqq & C^{\left|\bar{\Lambda}_{2}\right|} \prod_{x \in \Lambda_{2} \backslash I}\left\|\beta_{2}^{\#_{x}}\right\|_{2, p} \prod_{x \in I}\left\|\beta_{2}^{\#_{x}}\right\|_{r} \prod_{x \in\left[\left(\bar{\Lambda}_{2} \backslash \Lambda_{2}\right) \backslash E_{I}\right]}\left\|\beta_{1}^{\#_{x}}\right\|_{1, q} \prod_{x \in E_{I}}\left\|\beta_{1}^{\#_{x}}\right\|_{1,2} \\
& \cdot \prod_{\langle x, w\rangle \in \Gamma, x \in \bar{\Lambda}_{2}}\left\|\$(x, w)\left(e^{i \varphi(x) \cdot \varphi(w)}-b(x, w)\right) \chi\left(\varepsilon^{-\delta} \varphi(x)^{2}\right) \tilde{\chi}\left(\varepsilon^{-\delta} \varphi(w)^{2}\right)\right\|_{\infty},
\end{aligned}
$$

where $I=\left\{x \in \Lambda_{2} \mid \beta_{2}^{\#_{x}}=\varphi_{j} \beta_{2}\right.$ or $\left.\varphi_{j} \beta_{2}^{\prime}, j=1,2\right\}, E_{I}$ is a subset of $\bar{\Lambda}_{2} \backslash \Lambda_{2}$ with $\left|E_{I}\right| \leqq|I|$, and $\|\quad\|_{p_{1}, p_{2}}=\frac{1}{2}\left\{\|\|_{p_{1}}+\|\|_{p_{2}}\right\}$.

The proof of this lemma will be postponed to Subsect. 3.6.

Lemma 3.1 and the $L^{p}$ bounds of Subsect. 3.2 allow us to estimate $K$ in terms of $\varepsilon$ and $\left|\Lambda_{2}\right|$.

Lemma 3.2. For $\delta>0$ sufficiently small there exists $\delta_{1}>0$, depending only on the dimension $d$, such that for all $\varepsilon>0$ sufficiently small we have

$$
|K| \leqq \varepsilon^{\delta_{1}\left|\bar{\Lambda}_{2}\right|} \prod_{\langle x, w\rangle \in \Gamma, x \in \bar{\Lambda}_{2}}\left\|\$(x, w)\left(e^{i \varphi(x) \cdot \varphi(w)}-b(x, w)\right) \tilde{\chi}\left(\varepsilon^{-\delta} \varphi(x)^{2}\right) \tilde{\chi}\left(\varepsilon^{-\delta} \varphi(w)^{2}\right)\right\|_{\infty} .
$$


Proof. Let

$$
\begin{aligned}
& N_{1}=\left|\left[\bar{\Lambda}_{2} \backslash \Lambda_{2}\right] \backslash E_{I}\right|, \\
& N_{2}=\mid\left\{x \in \Lambda_{2} \mid \beta_{2}^{\#_{x}} \text { is either } \beta_{2} \text { or } \varphi_{j} \beta_{2}\right\} \mid, \\
& N_{2}^{\prime}=\mid\left\{x \in \Lambda_{2} \mid \beta_{2}^{\#_{x}} \text { is either } \beta_{2}^{\prime} \text { or } \varphi_{j} \beta_{2}^{\prime}\right\} \mid .
\end{aligned}
$$

We recall $B^{\prime \prime}=\left\{x \in \Lambda_{2} \mid \beta_{2}^{\#_{x}}=\left[2 \varphi^{2}\right]^{-1} \varphi_{j} \beta_{2}\right\}$.

Applying Lemma 3.1 and the bounds (3.6)-(3.25) we get

$$
|K| \leqq C^{\left|\bar{\Lambda}_{2}\right|} \varepsilon^{(1-\delta) N_{2}} \varepsilon^{-\delta N_{2}^{\prime}} \varepsilon^{(1-\delta)\left|B^{\prime \prime}\right|} \varepsilon^{-(1-1 / q) N_{1}} \varepsilon^{-1 / 2\left|E_{I}\right|} A,
$$

where

$$
A=\prod_{\langle x, w\rangle \in \Gamma, x \in \bar{\Lambda}_{2}}\left\|\$(x, w)\left(e^{i \varphi(x) \cdot \varphi(w)}-b(x, w)\right) \tilde{\chi}\left(\varepsilon^{-\delta} \varphi(x)^{2}\right) \tilde{\chi}\left(\varepsilon^{-\delta} \varphi(w)^{2}\right)\right\|_{\infty} .
$$

Now using (3.32) and (3.33), $\left|N_{2}^{\prime}\right|+\left|B^{\prime \prime}\right|=\left|B^{\prime}\right|$, the fact that $|I| \leqq\left|B^{\prime \prime}\right|$ by construction, $\left|E_{I}\right| \leqq|I|, N_{1} \leqq\left|\bar{\Lambda}_{2}\right|,\left|N_{2}\right|+\left|B^{\prime}\right|=\left|\Lambda_{2}\right|$ and $\left|\bar{\Lambda}_{2}\right| \leqq(2 d+1)\left|\Lambda_{2}\right|$, we get

$$
\begin{aligned}
|K| & \leqq C^{\left|\bar{\Lambda}_{2}\right|} \varepsilon^{\left[\left(2 \cdot 7^{d}\right)^{-1}-\delta\left(1+7^{-d}\right)\right]\left|B^{\prime}\right|} \varepsilon^{(1-\delta) N_{2}} \varepsilon^{-\left(1-q^{-1}\right)\left|\bar{\Lambda}_{2}\right|} A \\
& \leqq C^{(2 d+1)\left|\Lambda_{2}\right|} \varepsilon^{\left[\left(2 \cdot 7^{d}\right)^{-1}-\delta\left(1+7^{-d}\right)-\left(1-q^{-1}\right)(2 d+1)\right]\left|B^{\prime}\right|} \varepsilon^{\left[1-\delta-\left(1-q^{-1}\right)(2 d+1)\right] N_{2}} A .
\end{aligned}
$$
small.

The lemma now follows by taking $q$ sufficiently close to one and $\delta$ sufficiently

We can now prove the convergence of the expansion (3.28), by estimating it uniformly in $\Lambda$ and in $|E|<E_{0}, 0 \leqq \eta \leqq 1$.

Theorem 3.3. For any $E_{0}<\infty$ we can choose $\delta>0$ in (3.2) for which there exists $\varepsilon_{0}>0$ such that the expansion (3.28) converges as $\Lambda \rightarrow \mathbf{Z}^{d}$, uniformly in $0<\varepsilon<\varepsilon_{0}$, $|E| \leqq E_{0}$ and $0 \leqq \eta \leqq 1$.

Notice that Theorem 1.5 (i) follows from Theorem 3.3.

Proof of Theorem 3.3. From (3.28) and (3.29) we have

$$
\begin{aligned}
-\varepsilon i G_{\varepsilon, \Lambda}(z)=\sum_{\Lambda_{2} \subset \Lambda} & \sum_{\Lambda \in \mathscr{G}\left(\Lambda_{2}\right)} \sum_{\#, \$, b}( \pm) \int_{x \in\left[\left(\Gamma \cup \bar{\Lambda}_{2}\right) \backslash \Lambda_{2}\right]} \beta_{1}^{\# x}\left(\left(\varphi(x)^{2}\right) \prod_{x \in \Lambda_{2}} \beta_{2}^{\# x}\left(\varphi(x)^{2}\right)\right. \\
& \cdot \prod_{\langle x, y\rangle \in \Gamma} \$(x, y)\left(e^{i \varphi(x) \cdot \varphi(y)}-b(x, y)\right) \\
& \cdot \prod_{\langle x, y\rangle \in \bar{\Lambda}_{2}} \$(x, y) e^{i \varphi(x) \cdot \varphi(y)} \mathscr{D}_{\Gamma \cup \bar{\Lambda}_{2}} \varphi,
\end{aligned}
$$

where the sum in \#, $\$, b$ is over the allowable choice (3.35).

Using (3.36) we have

$$
\begin{aligned}
\left|\varepsilon G_{\varepsilon, \Lambda}(z)\right| \leqq & \sum_{\Lambda_{2} \subset \Lambda} \sum_{\Gamma \in \mathscr{G}_{2}(\Lambda)} \sum_{\#, \$, b}\left|K\left(\Lambda_{2}, \Gamma, \#, \$, b\right)\right| \\
& \cdot \prod_{\substack{\langle x, y\rangle \in \Gamma \\
x, y \notin \Lambda_{2}}}\left\|\$(x, y)\left(e^{i \varphi(x) \cdot \varphi(y)}-b(x, y)\right) \tilde{\chi}\left(\varepsilon^{-\delta} \varphi(x)^{2}\right) \tilde{\chi}\left(\varepsilon^{-\delta} \varphi(y)^{2}\right)\right\|_{\infty} \\
& \cdot \prod_{x \in \Gamma \backslash \bar{\Lambda}_{2}}\left\|\beta_{1}^{\# x}\right\|_{1} .
\end{aligned}
$$

For fixed $\Lambda_{2}, \Gamma, \#, \$, b$, let us write 


$$
\begin{aligned}
\Gamma_{1} & =\{\langle x, y\rangle \in \Gamma \mid b(x, y)=1\}, \\
L & =\left\{x \in \Gamma \backslash \bar{\Lambda}_{2} \mid \beta_{1}^{\#_{x}}=\beta_{1}\right\}, \\
L^{\prime} & =\left\{x \in \Gamma \backslash \bar{\Lambda}_{2} \mid \beta_{1}^{\#_{\mathrm{r}}}=\beta_{1}^{\prime}\right\} .
\end{aligned}
$$

We now choose $\delta>0$ and $\varepsilon_{0}>0$ by Lemma 3.2, so (3.38) holds for all $0 \leqq \varepsilon<\varepsilon_{0}$. Using (3.38), (3.6), (3.7) and (3.26) we have

$$
\left|\varepsilon G_{\varepsilon, \Lambda}(z)\right| \leqq \sum_{\Lambda_{2} \subset \Lambda} \sum_{\Gamma \in \mathscr{G}\left(\Lambda_{2}\right)} \sum_{\#, \$, b} \varepsilon^{\delta_{1}\left|\bar{\Lambda}_{2}\right|}(C \varepsilon|\log \varepsilon|)^{|L|}(C|\log \varepsilon|)^{\left|L^{\prime}\right|}\left(C \varepsilon^{\delta}\right)^{b},
$$

where $b_{\Gamma_{1}}$ is the number of bonds in $\Gamma_{1}$.

By the remark after (3.30) we have $\left|L^{\prime}\right| \leqq b_{\Gamma_{1}}$. Thus

$$
\begin{aligned}
\left|\varepsilon G_{\varepsilon, \Lambda}(z)\right| & \leqq \sum_{\Lambda_{2} \subset \Lambda} \sum_{\Gamma \in \mathscr{G}\left(\Lambda_{2}\right)} \sum_{\#, \$, b} \varepsilon^{\delta_{1}\left|\bar{\Lambda}_{2}\right|}(C \varepsilon|\log \varepsilon|)^{|L|}\left(C \varepsilon^{\delta / 2}|\log \varepsilon|\right)^{\left|L^{\prime}\right|} \\
& \leqq \sum_{\Lambda_{2} \subset \Lambda} \sum_{\Gamma \in \mathscr{G}\left(\Lambda_{2}\right)} \sum_{\#, \$, b} \varepsilon^{\delta_{2}\left|\Gamma \cup \bar{\Lambda}_{2}\right|}
\end{aligned}
$$

for some $\delta_{2}>0$.

We now estimate the sum on the right-hand side of (3.40) by first choosing the set $D=\Gamma \cup \bar{\Lambda}_{2}$, which must be a connected set containing the origin, and then summing over all possible choices of $\Lambda_{2}, \Gamma, \#, \$, b$ compatible with $D$. The number of possible choices is bounded by $C^{|D|}$ for some finite constant $C$ which depends only on the dimension. Thus we get

$$
\left|\varepsilon G_{\varepsilon, \Lambda}(z)\right| \leqq \sum_{0 \in D \text { connected }} C^{|D|} \varepsilon^{\delta_{2}|D|}=\sum_{n=1}^{\infty}(2 d)^{2 n}\left(C \varepsilon^{\delta_{2}}\right)^{n}=\sum_{1}^{\infty}\left(4 d^{2} C \varepsilon^{\delta_{2}}\right)^{n} .
$$

It suffices thus to choose $\varepsilon_{0}>0$ such that $4 d^{2} C \varepsilon_{0}^{\delta_{2}}<1$.

This finishes the proof of Theorem 3.3.

3.6. Proof of Lemma 3.1. At each site $x \in \Lambda_{2}$ we consider a function $f_{x}(\varphi(x))$ and define

$$
M\left(f_{x} ; x \in \bar{\Lambda}_{2}\right)=\int \prod_{x \in \bar{\Lambda}_{2}} f_{x}(\varphi(x)) \prod_{\langle x, y\rangle \in \bar{\Lambda}_{2}} \$(x, y) e^{i \varphi(x) \cdot \varphi(y)} \mathscr{D}_{\bar{\Lambda}_{2}} \varphi .
$$

$M\left(f_{x} ; x \in \bar{\Lambda}_{2}\right)$ is a multilinear functional. We will establish bounds for $M$ in terms of several possible choices of norms for the $\left\{f_{x} ; x \in \bar{\Lambda}_{2}\right\}$ and interpolate between these norms to obtain the desired bound that implies (3.36).

We start with some definitions. Let $J$ be the set of isolated points in $\Lambda_{2}$, i.e.,

$$
J=\left\{x \in \Lambda_{2} \mid\langle x, y\rangle \in \bar{\Lambda}_{2} \text { implies } y \in \Lambda_{1}\right\},
$$

and, for $\alpha$ as in (3.31), let

$$
H_{\alpha}=\left[\Lambda_{2} \cap\left(7 \mathbf{Z}^{d}+\alpha\right)\right] \backslash I,
$$

where $I$ is a given fixed subset of $\Lambda_{2}$ with $|x-y| \geqq 5$ for $x, y \in I, x \neq y$. For each $x \in I$ such that there exists $\langle x, y\rangle \in \bar{\Lambda}_{2}$ with $y \in \bar{\Lambda}_{2} \backslash \Lambda_{2}$, we choose such a $y$ which we denote by $y(x)$. We define

$$
E_{I}=\left\{y(x) \mid x \in I \quad \text { with } \operatorname{dist}\left(x, \bar{\Lambda}_{2} \backslash \Lambda_{2}\right)=1\right\} .
$$

Notice that $\left|E_{I}\right| \leqq|I|$.

We first prove: 
Lemma 3.4. For any $r, 2<r<\infty$, there exists $p, 1<p \leqq q$, where $1 / q=\frac{1}{2}+1 / r$, such that

$$
\begin{aligned}
& \left|M\left(f_{x} ; x \in \bar{\Lambda}_{2}\right)\right| \leqq C^{\left|\bar{\Lambda}_{2}\right|} \prod_{x \in H_{\alpha} \backslash J}\left\|f_{x}\right\|_{2} \prod_{x \in H_{\alpha} \cap J}\left\|f_{x}\right\|_{p} \prod_{x \in I}\left\|f_{x}\right\|_{r} \\
& \quad \cdot \prod_{x \in \Lambda_{2} \backslash\left(H_{\alpha} \cup I \cup J\right)}\left\|f_{x}\right\|_{1,2} \prod_{x \in \bar{\Lambda}_{2} \backslash\left(\Lambda_{2} \cup E_{I}\right)}\left\|f_{x}\right\|_{1, q} \prod_{x \in J \backslash H_{\alpha}}\left\|f_{x}\right\|_{1} \prod_{x \in E_{I}}\left\|f_{x}\right\|_{1,2} .
\end{aligned}
$$

Proof. We will first integrate over some of the variables in (3.42) and then estimate what remains by the $L^{1}$-norm. The integration will be done in several steps.

Step 1. Integration over the variables labeled by $J \cap H_{\alpha}$ and one nearest neighbor.

Since the sites in $J \cap H_{\alpha}$ do not have nearest neighbors in common we can do the integration over the corresponding variables obtaining

$$
\prod_{x \in J \cup H_{\alpha}} \hat{f}_{x}\left(\sum_{y:\langle x, y\rangle \in \bar{\Lambda}_{2}} \varphi(y)\right)
$$

where $\hat{f}(\varphi)=\int e^{i \varphi \cdot \varphi^{\prime}} f\left(\varphi^{\prime}\right) d^{2} \varphi^{\prime}$. We recall the Hausdorff-Young inequality $\|\hat{f}\|_{p^{\prime}} \leqq$ $(2 \pi)^{2-2 / p}\|f\|_{p}$ for $1 \leqq p \leqq 2,1 / p^{\prime}+1 / p=1$. Notice that if $x \in J \cap H_{\alpha}$ and we fix $\bar{y} \in \bar{\Lambda}_{2},|x-\bar{y}|=1$, then (3.44) is in $L^{p^{\prime}}\left(d^{2} \varphi(\bar{y})\right)$, uniformly in the other variables. Thus

$$
\int\left|f_{\bar{y}}(\varphi(\bar{y}))\right|\left|\hat{f}_{x}\left(\sum_{y:\langle x, y\rangle \in \bar{\Lambda}_{2}} \varphi(y)\right)\right| d^{2} \varphi(\bar{y}) \leqq 2 \pi\left\|f_{\bar{y}}\right\|_{p}\left\|f_{x}\right\|_{p} .
$$

Step 2. Integration over the variables labeled by $x \in H_{\alpha} \backslash J$ such that $\operatorname{dist}(x, I)>1$ and over one nearest neighbor.

For each such $x$ we pick $z=z(x) \in \Lambda_{2} \backslash H_{\alpha},|z-x|=1$. We then integrate over $\varphi(z)$, obtaining $\hat{f}_{z}\left(\sum_{y:\langle y, z\rangle \in \bar{\Lambda}_{2}} \varphi(y)\right)$, which is in $L^{2}\left(d^{2} \varphi(x)\right)$ uniformly in the other nearest neighbors of $z$. We obtain

$$
\int\left|f_{x}(\varphi(x))\right|\left|\hat{f}_{z}\left(\sum_{y:\langle y, z\rangle \in \bar{\Lambda}_{2}} \varphi(z)\right)\right| d^{2} \varphi(x) \leqq 2 \pi\left\|f_{x}\right\|_{2}\left\|f_{z}\right\|_{2} .
$$

Step 3. Integration over the variables labeled by $I$ and two nearest neighbors.

Here we must consider several cases.

Case 1. $x \in I$ and there is $y \in H_{\alpha}$ with $|x-y|=1$ (in particular $y \notin J$ ).

We integrate over $\varphi(y)$ to obtain $\hat{f}_{y}\left(\sum_{z:\langle z, y\rangle \in \bar{\Lambda}_{2}} \varphi(z)\right)$, which is in $L^{2}\left(d^{2} \varphi(x)\right)$ uniformly in the other variables. We then choose another nearest neighbor $z$ of $x$, which must be either in $\bar{\Lambda}_{2} \backslash \Lambda_{2}$ or in $\Lambda_{2} \backslash H_{\alpha}$. Integrating over $\varphi(z)$ we get $\hat{f}_{z}\left(\sum_{w:\langle w, z\rangle=1} \varphi(w)\right)$ which is in $L^{t}\left(d^{2} \varphi(x)\right)$, where $1 / t=1-1 / r-\frac{1}{2}$, uniformly in the other variables; here we pick an $r, 2<r<\infty$. We set $1 / q=1 / r+\frac{1}{2}$. We can then bound the integration over $\varphi(x)$ by $(2 \pi)^{2}\left\|f_{x}\right\|_{r}\left\|f_{y}\right\|_{2}\left\|f_{z}\right\|_{q}$.

Case 2. $x \in I$, $\operatorname{dist}\left(x, H_{\alpha}\right)>1$ but $\operatorname{dist}\left(x, \bar{\Lambda}_{2} \backslash \Lambda_{2}\right)=1$.

We pick $y(x) \in E_{I}$ and another nearest neighbor $z$ of $x$ and repeat the procedure of Case 1. We must now be careful if there exists $w \in H_{\alpha}$ such that $w$ and $x$ have a 
common nearest neighbor $u$. If in step 2 we chose a different nearest neighbor $u^{\prime}$ of $w$ over which to do the integration we can take $z=u$. If however we already used $u$ in step 2 we must take $z \neq u$. This can always be done except if we are in dimension $d=2$ and $x$ is a corner of the box $\Lambda$, so $x$ has only two nearest neighbors in $\Lambda$. In this case we must enlarge $E_{I}$ slightly (by four more points, one for each corner of $\Lambda$ ) and use this new site of $E_{I}$ in step 2 if necessary to free the two nearest neighbors of $x$ for the present case. This slight enlargement of $E_{I}$ makes no appreciable difference and we will simply ignore it.

Case 3. $x \in I$, dist $\left(x, H_{\alpha}\right)>1$, $\operatorname{dist}\left(x, \bar{\Lambda}_{2} \backslash \Lambda_{2}\right)>1$.

We proceed as in case 2 , but we pick the two nearest neighbors of $x$ in $\Lambda_{2} \backslash H_{\alpha}$.

Step 4. We now estimate all the remaining integrals by the $L^{1}$-norms.

If we now pick $p$ in step 1 such that $1<p \leqq q$, where $q$ was chosen in step 3, we obtain (3.43).

This proves Lemma 3.4 .

We will now use complex interpolation between Banach spaces to interpolate between the bounds obtained in Lemma 3.4 for different choices of $\alpha$. An application of the three lines theorem as in [25] gives

Lemma 3.5. Let $L\left(f_{x} ; x \in A\right)$ be a multilinear functional on $\left(L^{1} \cap L^{\infty}\right)^{A}$ such that

$$
\left|L\left(f_{x} ; x \in A\right)\right| \leqq C \prod_{x \in A}\left\|f_{x}\right\|_{t_{x}, u_{x}}
$$

and

$$
\left|L\left(f_{x} ; x \in A\right)\right| \leqq C \prod_{x \in A}\left\|f_{x}\right\|_{t_{x}, v_{x}}
$$

where $t_{x}, u_{x}, v_{x} \in[1, \infty]$.

Then

$$
\left|L\left(f_{x} ; x \in A\right)\right| \leqq C \prod_{x \in A}\left\|f_{x}\right\|_{t_{x}, w_{x}}
$$

where

$$
\frac{1}{w_{x}}=\frac{1}{2}\left(\frac{1}{u_{x}}+\frac{1}{v_{x}}\right) .
$$

We are now ready to finish the proof of Lemma 3.1. Let $\alpha^{(n)}, n=1,2, \ldots, 7^{d}$ denote an ordering of the $\alpha$ 's as in (3.31). We apply Lemma 3.5 to $M\left(f_{x} ; x \in \bar{\Lambda}_{2}\right)$, where we have $A=\Lambda_{2} \backslash I$ and fix the variables in $\bar{\Lambda}_{2} \backslash A$. We apply Lemma 3.5 iteratively. We start by choosing $\alpha=\alpha^{(1)}$ in Lemma 3.4 so we have the bounds (3.45) and (3.46) with

$$
\begin{aligned}
& t_{x}=2 \text { for } x \in \Lambda_{2} \backslash(I \cup J) \text {, } \\
& t_{x}=p \text { for } x \in J \text {, } \\
& u_{x}=1 \text { for } x \in \Lambda_{2} \backslash\left(H_{\alpha^{(1)}} \cup I\right) \text {, } \\
& u_{x}=p \text { for } x \in H_{\alpha^{(1)}} \text {, } \\
& v_{x}=1 \text { for } x \in A \text {. }
\end{aligned}
$$


Applying Lemma 3.5 we get (3.47). In the next step we take $\alpha=\alpha^{(2)}$ in Lemma 3.4, and use Lemma 3.5 with $t_{x}, u_{x}$ as before but with $v_{x}$ equal to $w_{x}$ obtained in the previous step. If we perform this procedure for all $\alpha^{(n)}, n=1, \ldots, 7^{d}$, we are able to obtain an estimate of the type (3.47) with all $w_{x}>1$ for all $x \in \Lambda_{2} \backslash I$. If we now select $p$ to be the smallest of all $w_{x}$ we obtain the estimate of Lemma 3.1.

3.7. Differentiation. Since $(d / d z) \beta\left(\Phi^{2} ; z\right)=i \Phi^{2} \beta\left(\Phi^{2} ; z\right)$, it follows from (3.1) that for $\operatorname{Im} z>0$ we have

$$
\frac{d^{n}}{d z^{n}} G_{\varepsilon, \Lambda}(z)=i \varepsilon^{-(n+1)} \sum_{x_{1}, \ldots, x_{n} \in \Lambda}\left\langle\psi(0) \bar{\psi}(0) \Phi\left(x_{1}\right)^{2} \ldots \Phi\left(x_{n}\right)^{2}\right\rangle_{\varepsilon, \Lambda},
$$

where

$$
\begin{aligned}
& \left\langle\psi(0) \bar{\psi}(0) \Phi\left(x_{1}\right)^{2} \ldots \Phi\left(x_{n}\right)^{2}\right\rangle_{\varepsilon, \Lambda} \\
& \quad=\int \psi(0) \bar{\psi}(0) \Phi\left(x_{1}\right)^{2} \ldots \Phi\left(x_{n}\right)^{2} \prod_{x \in \Lambda} \beta_{\varepsilon}\left(\Phi(x)^{2} ; x\right) \exp \left\{i \sum_{\langle x, y\rangle \in \Lambda} \Phi(x) \cdot \Phi(y)\right\} \mathscr{D}_{\Lambda} \Phi .
\end{aligned}
$$

We will now show that if $h$ is $2 n$-times differentiable with $(1+t) h^{(i)}(t)$ bounded, $i=0,1, \ldots, 2 n$, we can apply our modified cluster expansion to each term of the form (3.49) and obtain convergence of the expansion we get from (3.48) in the same region of the parameters for which we proved convergence for the expansion of $G_{\varepsilon, \Lambda}(z)$ (i.e., for the parameters as in Theorem 3.3). Since for fixed $E_{0}<\infty$ we will choose $\varepsilon_{0}>0$ independent of $n$, we will obtain a proof of Theorem 1.5 (ii) and (iii).

Applying the modified cluster expansion (3.28) to each term of the form (3.49), we get (we surpress $\varepsilon$ )

$$
\begin{aligned}
& \left\langle\psi(0) \bar{\psi}(0) \Phi^{2}\left(x_{1}\right) \ldots \Phi^{2}\left(x_{n}\right)\right\rangle_{\Lambda} \\
& \quad=\sum_{\Lambda_{2} \subset \Lambda} \sum_{\Gamma \in \mathcal{G}\left(\Lambda_{2} ; x_{1}, \ldots, x_{n}\right)}\left\langle\psi(0) \bar{\psi}(0) \Phi\left(x_{1}\right)^{2} \cdots \Phi\left(x_{n}\right)^{2}\right\rangle_{\Gamma, \Lambda_{2}},
\end{aligned}
$$

where

$$
\begin{aligned}
\langle A\rangle_{\Gamma, \Lambda_{2}}= & \int A \prod_{x \in \Lambda_{2}} \beta_{2}\left(\Phi(x)^{2}\right) \prod_{x \in\left[\left[\bar{A}_{2} \cup \Gamma \backslash \backslash \Lambda_{2}\right]\right.} \beta_{1}\left(\Phi(x)^{2}\right) \\
& \cdot \prod_{\langle x, y\rangle \in \Gamma}\left(e^{i \Phi(x) \cdot \Phi(y)}-1\right) \prod_{\langle x, y\rangle \in \bar{\Lambda}_{2}} e^{i \Phi(x) \cdot \Phi(y)} \mathscr{D}_{\Gamma \cup \bar{\Lambda}_{2}}
\end{aligned}
$$

and

$$
\mathscr{G}\left(\Lambda_{2} ; x_{1} \ldots x_{n}\right)=\left\{\Gamma \in \mathscr{G}\left(\Lambda_{2}\right) \mid x_{1}, \ldots, x_{n} \in \Gamma \cup \bar{\Lambda}_{2}\right\} .
$$

It now follows from (3.48) and (3.50) that

$$
\begin{aligned}
-i \varepsilon^{n+1} \frac{d^{n}}{d z^{n}} G_{\varepsilon, \Lambda}(z)= & \sum_{k=0}^{n} \sum_{x_{1}, \ldots, x_{n} \in \Lambda} \sum_{\Lambda_{2} \subset \Lambda} \sum_{\Gamma \in \mathscr{G}\left(\Lambda_{2} ; x_{1}, \ldots, x_{n}\right)}\left(\begin{array}{l}
n \\
k
\end{array}\right) \\
& \cdot\left\langle\psi(0) \bar{\psi}(0) \prod_{i=1}^{k} \bar{\psi}\left(x_{i}\right) \psi\left(x_{i}\right) \prod_{j=k+1}^{n} \varphi\left(x_{j}\right)^{2}\right\rangle_{\Gamma, \Lambda_{2}} .
\end{aligned}
$$

If we now perform the integration over the anticommuting variables in each 
term on the right-hand side of (3.51), we obtain a sum of terms of the same form as (3.29), except that:

(i) For $x=x_{1}, x_{2}, \ldots, x_{k}$, we have $\beta_{i}^{\# x}=\beta_{i}, i=1,2$.

(ii) For $x=x_{k+1}, \ldots, x_{n}, \beta_{i}^{\# x}$ is replaced by $\varphi^{2 m} \beta_{i}^{\# x}$, where $m$ denotes the number of $x_{j}$ 's, $j=k+1, \ldots, m$, such that $x=x_{j}$.

Since we did not assume that $\varphi^{2 m} \beta_{i}^{\#} \in L^{2}$, we will have to perform integrations by parts to obtain terms we can bound. In addition to the bounds of Subsect. 3.2, we will need:

$$
\begin{gathered}
\left\|\left|\varphi^{m}\right| \beta_{1}\right\|_{q} \leqq \varepsilon^{1 / 2 \delta m}\left\|\beta_{1}\right\|_{q} \\
\left\||\varphi|^{m} \beta_{1}^{(l)}\right\|_{q} \leqq \varepsilon^{1 / 2 \delta m}\left\|\beta_{1}^{(l)}\right\|_{q} .
\end{gathered}
$$

These bounds hold for all $1 \leqq q \leqq \infty$ and all $m \geqq 0$. Also,

$$
\left\||\varphi| \beta_{2}^{(l)}\right\|_{\infty} \leqq C \varepsilon^{-l+1-\delta / 2} \text {. }
$$

Since we assumed the same decay for $h^{(l)}$ as for $h$, we have that $\left\|\beta_{i}^{(l)}\right\|_{q}$ and $\varepsilon^{-l}\left\|\beta_{i}\right\|_{q}$ satisfy the same type of bounds, with $i=1,2$, with perhaps different constants.

Notice that the bounds (3.52) imply that for the terms in (3.51) with $x_{i} \in \Lambda \backslash \Lambda_{2}$ our previous estimates suffice. Problems arise from $x_{i} \in \Lambda_{2}$. We will show that such terms may, however also be estimated in essentially the same way as before, the bounds being changed by constants depending only on $n$ and $\varepsilon$.

For fixed $\Lambda_{2}$ and $\Gamma$ we consider a rather general term,

$$
\begin{aligned}
Z(\#, m, l) \equiv & Z\left(\#, m, l, \Lambda_{2}, \Gamma, \$, b\right) \\
= & \int \prod_{x \in \bar{\Lambda}_{2}}\left[\beta_{x}^{\# x}\left(\varphi(x)^{2}\right) \varphi_{1}^{m_{x}}(x) \varphi_{2}^{l_{x}}(x)\right] \prod_{\langle x, y\rangle \in \bar{\Lambda}_{2}} \$(x, y) e^{i \varphi(x) \cdot \varphi(y)} \\
& \cdot \prod_{\substack{\langle x, w\rangle \in \Gamma \\
x \in \bar{\Lambda}_{2}}} \$(x, w)\left[\varphi_{1}^{m_{w}}(w) \varphi_{2}^{l_{w}}(w) e^{i \varphi(x) \cdot \varphi(w)}-b(x, w)\right] \tilde{\chi}\left(\varepsilon^{-\delta} \varphi(w)^{2}\right) \mathscr{D}_{\bar{\Lambda}_{2}} \varphi .
\end{aligned}
$$

Here \#, $m, l$ are multi-indices taking values in the non-negative integers, and by $\beta_{x}^{\#_{x}}$ we mean the $\#_{x}$-th derivative of $\beta_{x}, \$$ and $b$ are the same as before, except that we will put $b(x, w)=0$ whenever $m_{w}+l_{w} \neq 0$. It is easy to see that $Z(\#, m, l)$ can be estimated as in the previous sections provided

(i) $l_{x}+m_{x} \leqq 1$ for all $x \in \Lambda_{2}$.

(ii) The distance between points with $l_{x}+m_{x}=1$ is sufficiently large, e.g., bigger than 6 in each coordinate direction.

Our purpose is to rewrite $Z(\#, m, l)$ as a sum of terms satisfying these conditions. This is achieved by using partial integration to move around powers of $\varphi$ until they are sufficiently diluted. The key to success is that the number of terms generated this way depends only on the number of powers of $\varphi$ originally present, i.e. on $n$, and that the bounds on the resulting terms differ from the bounds obtained previously only by factors depending on $\varepsilon$ and $n$. The following describes a simple algorithm to achieve this. Note that we do not strive for optimal bounds. We use 
the following notation: $|m|=\sum_{x \in \bar{\Lambda}_{2}} m_{x},|l|,|\#|$ being defined the same way. Let $\pi$ be a fixed (lattice) hyperplane intesecting the boundary of $\Lambda$. For $x \in \Lambda$, let $d(x) \equiv$ $\operatorname{dist}(\pi, x)$. We denote by $\pi_{\rho}$ a (hyper) plane parallel to $\pi$ such that $\operatorname{dist}\left(\pi, \pi_{\rho}\right)=\rho$. We are only concerned with $\pi_{\rho}$ 's that intersect $\Lambda_{2}$. For any such plane let

$$
\sigma_{\rho}(m, l)=\sum_{x \in \Lambda_{2} \cap \pi_{\rho}} m_{x}+l_{x}
$$

A plane $\pi_{\rho}$ with $\sigma_{\rho}(m, l)=0$ is called "empty" and a plane with $\sigma_{\rho}(m, l)=1$ is called "clean." We will rewrite $Z(\#, m, l)$ as a sum of similar terms in which all planes will become clean or empty, and such that between two clean planes there will be at least six empty ones. Let

$$
\begin{gathered}
\mathscr{C}(m, l)=\left\{x \in \Lambda_{2} \mid \text { either } \sigma_{d(x)}(m, l)>1, \text { or } \sigma_{d(x)}(m, l)=1 \text { and } \sigma_{d^{\prime}}(m, l) \neq 0\right. \\
\text { for some } \left.d^{\prime} \text { with }\left|d(x)-d^{\prime}\right| \leqq 6\right\}
\end{gathered}
$$

and

$$
\Delta(m, l)=\sum_{x \in \mathscr{C}(m, l)} m_{x}+l_{x}
$$

The following lemma provides the elementary operation we need:

Lemma 3.6. Let $x_{0} \in \Lambda_{2}$. Let $x_{1}$ be the nearest neighbor of $x_{0}$ such that $d\left(x_{1}\right)=d\left(x_{0}\right)-1$. Let $N_{1}$ be the set of nearest neighbors of $x_{1}$ in $\bar{\Lambda}_{2} \cup \Gamma$, and $N_{1}^{0}=N_{1} \backslash\left\{x_{0}\right\}$. Then

$$
Z(\#, m, l)=\sum_{y \in N_{1}} Z(\#(y), m, l(y))+l_{x_{1}} Z\left(\#, m, l\left(x_{1}\right)\right)
$$

if $l_{x_{0}}>0$, and

$$
Z(\#, m, l)=\sum_{y \in N_{1}} Z(\#(y), m(y), l)+m_{x_{1}} Z\left(\#, m\left(x_{1}\right), l\right)
$$

if $m_{x_{0}}>0$, where

$$
\begin{aligned}
m_{x}(y)= & m_{x_{0}}-1 \text { if } x=x_{0}, \\
& m_{y}+1 \quad \text { if } x=y \in N_{1}^{0}, \\
& m_{x_{1}}+1 \text { if } x=x_{1} \text { and } y=x_{0}, \\
& m_{x_{1}}-1 \text { if } x=x_{1} \text { and } y=x_{1}, \\
& m_{x} \text { otherwise. }
\end{aligned}
$$

$l_{x}(y)$ is defined in the same way and

$$
\begin{gathered}
\#_{x}(y)=\#_{x_{1}}-1 \text { if } x=x_{1} \text { and } y=x_{0}, \\
\#_{x} \text { otherwise. }
\end{gathered}
$$

Proof. Consider the case $l_{x_{0}} \neq 0$. We may use in (3.55) the identity

$$
\varphi_{2}^{l_{x_{0}}}\left(x_{0}\right) e^{i \varphi\left(x_{0}\right) \cdot \varphi\left(x_{1}\right)}=\varphi_{2}^{l_{x_{0}}-1}\left(x_{0}\right)\left[\frac{\partial}{i \partial \varphi_{2}\left(x_{i}\right)} e^{i \varphi\left(x_{0}\right) \cdot \varphi\left(x_{1}\right)}\right] .
$$

Performing then an integration by parts with respect to the variable $\varphi_{2}\left(x_{1}\right)$ and 
computing the derivatives that arise we get

$$
\begin{aligned}
Z(\#, m, l)= & \int \prod_{x \neq x_{1}} \beta_{x}^{\# x}\left(\varphi(x)^{2}\right) \varphi_{1}^{m_{x}}(x) \varphi_{2}^{l_{x}}(x) \prod_{\langle x, y\rangle \in \bar{\Lambda}_{2}} \$(x, y) e^{i \varphi(x) \cdot \varphi(y)} \\
& \cdot \prod_{\substack{x, w\rangle \in \Gamma \\
x \in \bar{\Lambda}_{2}}} \$(x, w)\left[\varphi_{1}^{m_{w}}(w) \varphi_{2}^{l_{w}}(w) e^{i \varphi(x) \cdot \varphi(w)}\right. \\
& -b(x, w)] \tilde{\chi}\left[\varepsilon^{-\delta} \varphi(\mathrm{w})^{2}\right] \\
& \cdot \varphi_{2}\left(x_{0}\right)^{-1}\left\{\sum _ { y \in N _ { 1 } ^ { 0 } } \beta _ { x _ { 1 } } ^ { \# _ { x _ { 1 } } } \left(\varphi\left(x_{1}\right)^{2} \varphi_{1}^{m_{x_{1}}}\left(x_{1}\right) \varphi_{2}^{l_{x_{1}}}\left(x_{1}\right) \varphi_{2}(y)\right.\right. \\
& +\beta_{x_{1}}^{\#_{x_{1}}+1}\left(\varphi\left(x_{1}\right)^{2}\right) \varphi_{1}^{m_{x_{1}}}\left(x_{1}\right) \varphi_{2}^{l_{x_{1}}+1}\left(x_{1}\right) \\
& +l_{x_{1}} \beta_{x_{1}}^{\#_{x_{1}}}\left(\varphi\left(x_{1}\right)^{2} \varphi_{1}^{m_{x_{1}}}\left(x_{1}\right) \varphi_{2}^{l_{x_{1}}-1}\left(x_{1}\right)\right\} \mathscr{D}_{\bar{\Lambda}_{2}} \varphi
\end{aligned}
$$

Equation (3.61) is strictly correct only if $N_{1}^{0} \subset \bar{\Lambda}_{2}$. If $N_{1}^{0}$ contains points $y \in \Gamma$ but $y \notin \bar{\Lambda}_{2}$, the $b(x, y)$ corresponding to that term in the sum disappears. This is in accordance with our convention that $b(x, w)=0$ if $m_{w}+l_{w} \neq 0$. Taking this remark into account, Lemma 3.6 can be read off Eq. (3.61).

Note that the multi-indices appearing in Lemma 3.6 satisfy

$$
\begin{gathered}
|m|+|l(y)| \leqq|m|+|l|, \\
|m(y)|+|l| \leqq|m|+|l|, \\
\sigma_{\rho}(m(y), l)=\sigma_{\rho}(m, l(y))=\sigma_{\rho}(m, l) \quad \text { if } \quad \rho>d\left(x_{0}\right)=\sigma_{\rho}(m, l)-1 \\
\text { if } \rho=d\left(x_{0}\right),
\end{gathered}
$$

and

$$
|\#(y)| \leqq|\#|+1
$$

We may use Lemma 3.6 successively in a given plane $\pi_{\rho}$ until that plane is "clean" or "empty." Keeping track of the terms that are produced gives:

Lemma 3.7. Let $\pi_{\rho}$ be a plane such that $\sigma_{\rho}(m, l) \geqq 1$. Let $\tau=0$ or 1 .

Then

$$
Z(\#, m, l)=\sum_{i=1}^{\kappa} Z\left(\tilde{\#}_{i}^{\tau}, \tilde{m}_{i}^{\tau}, \tilde{l}_{i}^{\tau}\right)
$$

where

(i) $\sigma_{\rho}\left(\tilde{m}_{i}^{\tau}, \tilde{l}_{i}^{\tau}\right)=\tau$ for all $i$,

(ii) $\left|\tilde{m}_{i}^{\tau}\right|+\left|\tilde{l}_{i}^{\tau}\right| \leqq|m|+|l|$,

(iii) $\sigma_{\rho^{\prime}}\left(\tilde{m}_{i}^{\tau}, \tilde{l}_{i}^{\tau}\right)=\sigma_{\rho}(m, l)$ for $\rho^{\prime}>\rho$,

(iv) $\quad\left|\tilde{\#}_{i}^{\tau}\right| \leqq|\#|+\sigma_{\rho}(m, l)-\tau$,

(v) $\quad \#_{i x}^{\tau} \leqq \#_{x}+\sigma_{\rho}(m, l)-\tau$ for $x \in \pi_{\rho-1}$,

$$
=\#_{x} \quad \text { for } x \notin \pi_{\rho-1}
$$




$$
\kappa \leqq\left[2 d+\sigma_{\rho-1}(m, l)+\sigma_{\rho}(m, l)\right]^{\sigma_{\rho}^{(m, l)-\tau}} .
$$

Proof. The lemma follows by applying Lemma $3.6 \sigma_{\rho}(m, l)-\tau$ times.

We now apply Lemma 3.7 consecutively on non-empty planes starting with the one farthest away from $\pi$. In the first plane we choose $\tau=1$, thus leaving it "clean." In the subsequent 6 planes we apply, if necessary, Lemma 3.7 with $\tau=0$. The $7^{\text {th }}$ plane again is treated only until "clean" and so on. For each seven planes we treat we reduce thus $\Delta(m, l)$ by one. Therefore, the total number of times we need to apply the lemma is no larger than $7 \cdot 2 r$, if $\Delta(h, l)=2 r$ at the beginning, until $\Delta(\tilde{m}, \widetilde{l})=0$. But this guarantees that powers of $\varphi$ in $\Lambda_{2}$ that appear are all at most one, and furthermore widely separated. More precisely, we have

Proposition 3.8. Let $\bar{\Lambda}_{2}$ be fixed, $|m|+|l|=2 r$, and \# a multi-index with $\#_{x} \leqq 1$ for all $x \in \bar{\Lambda}_{2}$. Then

$$
Z(\#, m, l)=\sum_{i=1}^{\kappa} Z(\tilde{\#}(i), \tilde{m}(i), \tilde{l}(i))
$$

where

(i) $\Delta(\tilde{m}(i), \tilde{l}(i))=0$,

(ii) $|\tilde{m}(i)|+|\tilde{l}(i)| \leqq 2 r$,

(iii) $\quad|\tilde{\#}(i)| \leqq|\#|+7 \cdot(2 r)^{2}$,

(iv) $\quad \tilde{\#}_{x}(i) \leqq \tilde{\#}_{x}+2 r-1 \leqq 2 r$ for all $x$,

(v) $\quad \kappa \leqq(2 d+4 r)^{(2 r)^{2}}$.

Proof. Again, the proposition follows by using Lemma 3.7 as outlined above and keeping track of the bounds, using that in particular $\sigma_{\rho}(m, l) \leqq 2 r$ in each plane $\pi_{\rho}$ and at each stage of the process.

A term $Z(\tilde{\#}, \tilde{m}, \tilde{l})$ can now be estimated in \|\|$_{\infty}$-norm like a term $K$ in Lemma 3.1., after we perform integrations by part on a set $B^{\prime \prime} \subset B^{\prime}$. To avoid complications, we choose $B^{\prime \prime}$ as in Subsect. 3.4, but remove from it all points such that $\tilde{l}_{x}+\tilde{m}_{x}=1$. There are at most $2 r \cdot 2^{d}$ such points, and will therefore affect our bounds only by a factor $\varepsilon^{-2 r 2^{d}}$.

Taking this into account and using Lemma 3.2 with the bounds (3.52)-(3.54) gives then that

$$
\begin{aligned}
|Z(\tilde{\#}, m, \tilde{l})| \leqq \varepsilon^{-a(r)} \varepsilon^{\delta_{1}\left|\bar{\Lambda}_{2}\right|} & \cdot \prod_{\substack{\langle x, w\rangle \in \Gamma \\
x \in \bar{\Lambda}_{2}}}\left\|\$(x, w)\left(e^{i \varphi(x) \cdot \varphi(w)}-b(x, w)\right) \tilde{\chi}\left[\varepsilon^{-\delta} \varphi(x)^{2}\right] \tilde{\chi}\left[\varepsilon^{-\delta} \varphi(w)^{2}\right]\right\|_{\infty},
\end{aligned}
$$

where $a(r)$ is a constant depending only on $r$. [Note that $a(r)$ takes already into account the fact that some (but no more than $2 r$ ) terms in the product on the right of (3.67) appear modified in $Z(\tilde{\#}, \tilde{m}, \tilde{l})$.] Note that $\delta_{1}$ is the same that appears in Lemma 3.2. Combining this estimate with the bound on in Proposition 3.7, we 
get that

$$
\begin{gathered}
\left|-i \varepsilon^{n+1} \frac{d^{n}}{d z^{n}} G_{\varepsilon, \Lambda}(z)\right| \leqq 2^{n} \sum_{x_{1} \ldots x_{n} \in \Lambda} \sum_{\Lambda_{2} \backslash \Lambda} \sum_{\Gamma \in \mathscr{G}\left(\Lambda_{2} ; x_{1}, \ldots, x_{m}\right)} \sum_{\#, \$, b} \\
\cdot|Z(\#, l, m)| \prod_{\substack{\langle x, y\rangle \in \Gamma \\
x, y \notin \bar{\Lambda}_{2}}}\left\|\$(x, y)\left(e^{i \varphi(x) \cdot \varphi(y)}-b(x, y)\right) \tilde{\chi}\left(\varepsilon^{-\delta} \varphi(x)^{2}\right) \tilde{\chi}\left(\varepsilon^{-\delta} \varphi(y)^{2}\right]\right\|_{\infty} \\
\cdot \prod_{x \in \Gamma \backslash \bar{\Lambda}_{2}}\left\|\beta_{1}^{\# x}\right\|_{1} \leqq \sum_{\substack{0 \in D \\
\text { connected }}}|D|^{n} C_{1}(n) \varepsilon^{-a(n)} C^{|D|} \varepsilon^{\delta_{2}|D|} ;
\end{gathered}
$$

here $C_{1}(n), a(n)$ depend only on $n$, and $C, \delta_{2}$ are constants independent of $n$. The latter sum converges, as (3.41), provided $\varepsilon_{0}>\varepsilon>0$, if

$$
4 d^{2} C \varepsilon_{0}^{\delta_{2}} \leqq 1 \text {. }
$$

This proves Theorem 1.5 (ii). Since $\varepsilon_{0}$ given by (3.69) is independent of $n$, we proved Theorem 1.5 (iii) as well.

\section{References}

1. Benderskii, M., Pastur, L.: On the spectrum of the one dimensional Schrödinger equation with a random potential. Mat. Sb. 82, 245-256 (1970)

2. Avron, J., Simon, B.: Almost periodic Schrödinger operators. II. The integrated density of states. Duke Math. J. 50, 369-391 (1983)

3. Kirsch, W., Martinelli, F.: On the density of states of Schrödinger operators with a random potential. J. Phys. A15, 2139-2156 (1982)

4. Carmona, R.: Random Schrödinger Operators. Proceedings of the ecole d'ete de probabilite XIV, Saint-Flour, 1984. Lecture Notes in Mathematics, Vol. 1180. Berlin, Heidelberg, New York: Springer 1986

5. Pastur, L.: Spectral properties of disordered systems in one-body approximation. Commun. Math. Phys. 75, 179 (1980)

6. Craig, W., Simon, B.: Subharmonicity of the Lyaponov Index. Duke Math. J. 50, 551-560 (1983)

7. Delyon, F., Souillard, B.: Remark on the continuity of the density of states of ergodic finite difference operators. Commun. Math. Phys. 94, 289 (1984)

8. LePage, E.: Empirical distribution of the eigenvalues of a Jacobi matrix. In: Probability measures on groups, VII. Lecture Notes in Mathematics, Vol. 1064. Berlin, Heidelberg, New York: Springer 1983

9. Carmona, R., Klein, A., Martinelli, F.: Anderson localization for Bernoulli and other singular potentials. Commun. Math. Phys. 108, 41-66 (1987)

10. Simon, B., Taylor, M.: Harmonic analysis on $S L(2, R)$ and smoothness of the density of states in the one-dimensional Anderson model. Commun. Math. Phys. 101, 1-19 (1985)

11. Campanino, M., Klein, A.: A supersymmetric transfer matrix and differentiability of the density of states in the one-dimensional Anderson model. Commun. Math. Phys. 104, 227-241 (1986)

12. March, P., Sznitman, A.: Some connections between excursion theory and the discrete random Schrödinger equation, with applications to analyticity and smoothness properties of the density of states in one dimension. Preprint

13. Edwards, S., Thouless, D.: Regularity of the density of states in Anderson's localized electron model. J. Phys. C4, 453-457 (1971)

14. Wegner, F.: Bounds on the density of states in disordered systems. Z. Phys. B44, 9-15 (1981)

15. Maier, R.: Bounds on the density of states of random Schrödinger operators, preprint

16. Constantinescu, F., Fröhlich, J., Spencer, T.: Analyticity of the density of states and replica method for random Schrödinger operators on a lattice. J. Stat. Phys. 34, 571-596 (1984)

17. Simon, B.: Kotani theory for one dimensional stochastic Jacobi matrices. Commun. Math. Phys. 89, 227-234 (1983) 
18. Parisi, G., Sourlas, N.: Self avoiding walk and supersymmetry. J. Phys. Lett. 41, 2403-2406 (1980)

19. McKane, A. J.: Reformulation of $n \rightarrow 0$ models using anticommuting scalar fields. Phys. Lett. A76, 22-24 (1980)

20. Luttinger, J. M.: The asymptotic evaluation of a class of path integrals, II. J. Math. Phys. 24, 2070-2073 (1983)

21. Effetov, K. B.: Supersymmetry and the theory of disordered metals. Adv. Phys. 32, 53-127 (1983)

22. Klein, A., Martinelli, F., Perez, J. F.: A rigorous replica approach to Anderson localization in one dimension. Commun. Math. Phys. 106, 623-633 (1986)

23. Berezin, F. A.: The method of second quantization. New York: Academic Press 1966

24. Klein, A., Landau, L. J., Perez, J. F.: Supersymmetry and the Parisi-Sourlas dimensional reduction: A rigorous proof. Commun. Math. Phys. 93, 459-482 (1984)

25. Stein, E., Weiss, G.: Introduction to Fourier Analysis in Euclidean Spaces. Princeton: Princeton University Press 1971

Communicated by T. Spencer

Received July 8, 1987 
